Linköping Studies in Science and Technology

Dissertations No. 1781

\title{
Permanence of age-structured populations in a spatio-temporal variable environment
}

Sonja Radosavljevic

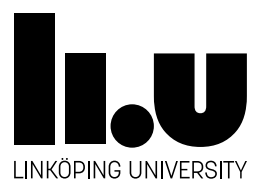

Department of Mathematics, Division of Mathematics and Applied Mathematics Linköping University, SE-581 83 Linköping, Sweden

Linköping 2016 
Linköping Studies in Science and Technology. Dissertations No. 1781

Permanence of age-structured populations in a spatio-temporal variable environment

Copyright $\odot$ Sonja Radosavljevic, 2016

Division of Mathematics and Applied Mathematics

Department of Mathematics

Linköping University

SE-581 83, Linköping, Sweden

sonja.radosavljevic@liu.se

ISSN 0345-7524

ISBN 978-91-7685-706-9

Printed by LiU-Tryck, Linköping, Sweden 2016 
To Aleksa,

whom I met somewhere along the road to Hyperborea... 



\section{Abstract}

It is widely recognized that various biotic and abiotic factors cause changes in the size of a population and its age distribution. Population structure, intra-specific competition, temporal variability and spatial heterogeneity of the environment are identified as the most important factors that, alone or in combination, influence population dynamics. Despite being well-known, these factors are difficult to study, both theoretically and empirically. However, in an increasingly variable world, permanence of a growing number of species is threatened by climate changes, habitat fragmentation or reduced habitat quality. For purposes of conservation of species and land management, it is crucially important to have a good analysis of population dynamics, which will increase our theoretical knowledge and provide practical guidelines.

One way to address the problem of population dynamics is to use mathematical models. The choice of a model depends on what we want to study or what we aim to achieve. For an extensive theoretical study of population processes and for obtaining qualitative results about population growth or decline, analytical models with various level of complexity are used. The competing interests of realism and solvability of the model are always present. This means that, on one hand, we always aim to set up a model that will truthfully reflect reality, while on the other hand, we need to keep the model mathematically solvable. This prompts us to carefully choose the most prominent ecological factors relevant to the problem at hand and to incorporate them into a model. Ideally, the results give new insights into population processes and complex interactions between the mentioned factors and population dynamics.

The objective of this thesis is to formulate, analyze, and apply various mathematical models of population dynamics. We begin with a classical linear age-structured model and gradually add temporal variability, intra-specific competition and spatial heterogeneity. In this way, every subsequent model is more realistic and complex than the previous one. We prove existence and uniqueness of a nonnegative solution to each boundary-initial problem and continue with investigation of the large-time behavior of the solution.

In the ecological terms, this means that we are establishing conditions under which a population can persist in a certain environment. Since our aim is a qualitative analysis of a solution, we often examine its upper and lower bounds. Their importance is in the fact that they are obtained analytically and parameters in their expression have biological meaning. Thus, instead of analyzing an exact solution (which often proves to be difficult), we analyze the corresponding upper and lower solutions.

We apply our models to demonstrate the influence of seasonal changes (or some other periodic temporal variation) and spatial structure of the habitat on population persistence. This is particularly important in explaining behavior of migratory birds or populations that inhabits several patches, some of which are of low quality. Our results extend the previously obtained results in some aspects and point out that all factors (age structure, density dependence, spatio-temporal variability) need to be considered in making a population model and predicting population growth. 


\section{Populärvetenskaplig sammanfattning}

Populationer i sina naturliga miljöer är ofta utsatta för olika biotiska och abiotiska faktorer som kan vara till nytta eller skada för befolkningstillväxten. Några av dessa faktorer är befolkningens åldersstruktur, konkurrens, temporala förändringar i omgivningen (såsom klimatförändringar, förändringar av säsongen eller dagliga förändringar i temperatur, solljus och nederbörd), rumslig heterogenitet och struktur av livsmiljön och mänsklig påverkan.

Enligt data kan vi se att ett växande antal arter hotas av mer frekventa och allvarliga förändringar i miljön. Detta kräver en omfattande teoretisk och empirisk studie av sambandet mellan förändringar i interna faktorer, omvärldsvariationen och populationsdynamik. Ett sätt att hantera detta problem teoretiskt är att använda analytiska matematiska modeller för populationsdynamik.

I den här avhandlingen presenterar vi flera matematiska modeller som beskriver populationstillväxten med olika nivåer av detalj. I det första steget bevisar vi existens och entydighet av en icke-negativ lösning till modellen. I det andra steget analyserar vi asymptotisk beteendet av en lösning.

Artikel I behandlar asymptotiken av den klassiska linjära åldersstrukturerade populationsmodell. Vi antar att befolkningen lever i en tidsmässigt föränderlig miljö och studerar effekter av variation på befolkningstillväxten. I Artikel II inkluderar vi konkurrens om resurser och får en icke-linjär populationsmodell med logistisk term. Detta ger oss möjlighet att studera en kombination av åldersstruktur, tidsberoende och densitetsberoende och deras inverkan på befolkningens stabilitet. Artikel III betraktar situationen när befolkningen lever i flera patchar. Lokala populationens dynamik förklaras med hjälp av resultat från Artikel II. Vi antar att spridning är möjlig mellan patcharna och bestämmer spridningsmönster som kommer att minska utdöenderisk. Vi använder också denna modell för att beskriva migration av fåglar och för att hitta när utdöende på alla patchar kommer att inträffa.

Från ett matematiskt perspektiv är befolkningsproblemet mer komplext i varje efterföljande papper. Att hitta en exakt lösning kan vara mycket utmanande. På grund av detta formulerar vi två extra problem: en beskriver den bästa situation för befolkningstillväxten och den andra beskriver den värsta situationen. Det är mycket enklare att lösa dessa hjälpproblem, och deras lösningar representerar övre och undre gränser för en lösning på det ursprungliga problemet. På detta sätt erhåller vi kvalitativ analys av asymptotiken av lösningen till det ursprungliga problemet.

Våra resultat visar att alla de interna och externa faktorerna har en viss effekt på befolkningstillväxten. De indikerar också sambandet mellan livshistorien och populationens svar på omvärldsvariationen. Vi tror att modellerna bidrar till vår förståelse av populationsdynamik och ökar vår förmåga att göra prognoser för befolkningstillväxt, som kan hjälpa oss att uppskatta utdöenderisken eller förbättra förvaltningsstrategier. 


\section{Acknowledgements}

First and foremost, I would like to express my sincere gratitude to my supervisor, Professor Vladimir Kozlov, for his continuous support, patience and guidance. He has always been able to point me to the right direction and helped me develop as a researcher, but more than that, he has been an invaluable source of knowledge and motivation all these years.

I am deeply indebted to my co-supervisor, Professor Uno Wennergren, who showed me the beauty of ecology and guided me through my scientific meanderings. He has been a constant and inexhaustible source of encouragement, knowledge and inspiration. More so, his enthusiasm has been contagious and lifted my spirit many times over the years.

I am thankful to my co-supervisor Bengt Ove Turesson for the given support, patience and advice.

I would like to extend my gratitude to Professor Vladimir Tkachev for fruitful discussions and inspiring collaboration. All help and kindness he gave along the way are highly appreciated.

Professor Dragan Djordjevic have my thanks for keeping me interested in mathematics and introducing me to the operator theory.

I would like to thank all of my colleagues at MAI for making a pleasant working environment. I am especially grateful to Jola, Anna, Spartak, Nisse, Samira, Arpan, Evgeny, Mikael, Leslie, Marcus and Alexandra and for their friendship. My thanks also goes to everyone at the Division of theoretical ecology for showing me a new world, all interesting talks, encouragement and kindness. 


\section{List of Papers}

The following papers are included in this thesis and will be referred to by their roman numerals.

I V. Kozlov, S. Radosavljevic, B. O. Turesson, U. Wennergren, Estimating effective boundaries of population growth in a variable environment, accepted to be published in the Boundary Value Problems

II V. Kozlov, S. Radosavljevic, U. Wennergren, Large time behavior of logistic agestructured population model, submitted

III V. Kozlov, S. Radosavljevic, V. Tkachev, U. Wennergren, Persistence analysis of the age-structured population model on several patches, Proceedings to the 16th international conference on computational and mathematical methods in science and engineering, CMMSE 2016, Costa Ballena, Cadiz, Spain, 4-8 July, 2016, 717-727

IV V. Kozlov, S. Radosavljevic, V. Tkachev, U. Wennergren, Permanence of age-structured population model on several temporally variable patches, manuscript

In all aforementioned works I have contributed by doing all detailed derivations, discussing the study problem and the results and writing the papers.

The papers that will not be included in thesis are as follows:

1. S. Radosavljevic and D. S. Djordjevic, On MP and Drazin inverse of product, difference, and sum of two projections on a Hilbert space, accepted to be published in Filomat

2. U.Akram, S. Radosavljevic, N-H. Quttineh, U. Wennergren. Managing locally available nutrients for ecosystem health and future food security: A spatial analysis in Sweden and Pakistan. Proceedings of the 2nd International Conference on Global Food Security, October 11-14, 2015, Ithaca, NY, USA.

3. S. Radosavljevic and D. S. Djordjevic, On pairs of generalized and hypergeneralized projections in a Hilbert space, Functional Analysis, Approximation and Computation 5:2 (2013), 67-75

4. S. Radosavljevic, Pairs of projections on a Hilbert space: properties and generalized invertibility, Licenciate thesis, Linköping university, 2012 


\section{Contents}

Abstract ........................ i

Populärvetenskaplig sammanfattning . . . . . . . . . . . . . . iii

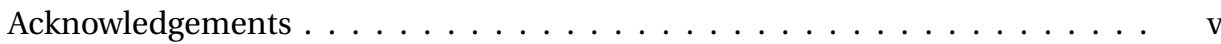

List of Papers $\ldots \ldots \ldots \ldots \ldots \ldots \ldots$ vii

$\begin{array}{ll}\text { Introduction } & 1\end{array}$

1 Modeling population dynamics 3

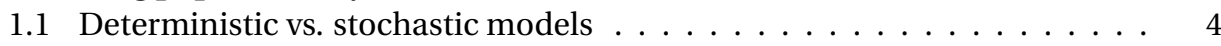

1.2 Organization of the thesis $\ldots \ldots \ldots \ldots \ldots$

2 Ecological considerations 6

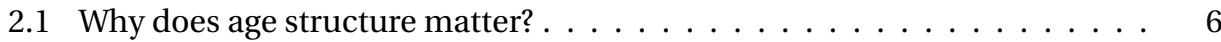

2.2 On the role of time $\ldots \ldots \ldots \ldots \ldots \ldots$

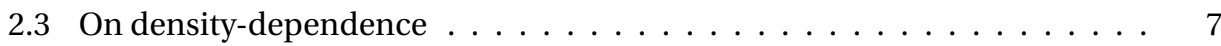

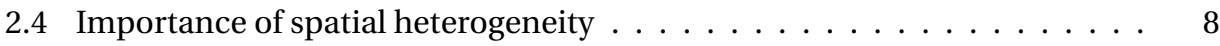

3 On linear age-structured population model in a variable environment 10

3.1 Constant environment . . . . . . . . . . . . . . . . . 10

3.2 Temporally varying environment . . . . . . . . . . . . . . . 12

3.3 Asymptotics of the linear age-structured time-dependent model . . . . . . . . . . . . . . . . . 12

3.3.1 General upper and lower bounds . . . . . . . . . . . . . . . . . 13

3.3.2 Upper and lower bounds through time-independent model . . . . . 14

3.3.3 Periodical variation of environment . . . . . . . . . . . . . . . . 15

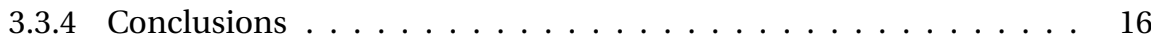

4 Logistic age-structured population model in a variable environment 18

4.1 Modeling density-dependence . . . . . . . . . . . . . . . 18

4.2 Age-structured model with logistic term . . . . . . . . . . . . . . . . . 18

4.2.1 Existence and uniqueness of a solution . . . . . . . . . . . . . . . 19

4.2.2 Asymptotics in a constant environment . . . . . . . . . . . . 20

4.2.3 Asymptotics in a variable environment . . . . . . . . . . . . . . 21

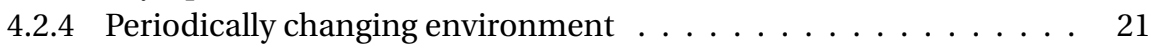

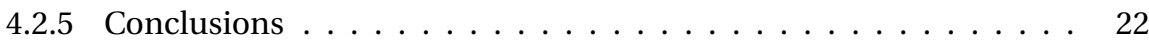

5 Age-structured population model in a

spatio-temporally varying environment $\quad 24$

5.1 Population in a patchy environment . . . . . . . . . . . . . . . . . . 24

5.2 Asymptotics in a constant environment . . . . . . . . . . . . . 26

5.2.1 Two-side estimates for $\sigma\left(\mathscr{R}_{0}\right)$ and for the solution to (5.9) . . . . . 28

5.3 Asymptotics in a periodic environment . . . . . . . . . . . . . . 29 
5.4 Two-side estimates in an irregular environment . . . . . . . . . . . . . . . . . . 30

5.5 Source-sink dynamics . . . . . . . . . . . . . . . . . 30

5.5 .1 A single source and several sinks . . . . . . . . . . . . . 30

5.5 .2 Sinks without a source . . . . . . . . . . . . . . 31

5.5 .3 Extinction on all patches . . . . . . . . . . . . 32

6 Discussion $\quad 34$

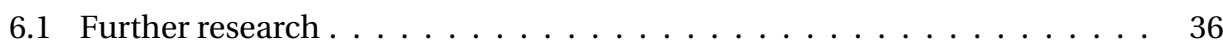

$\begin{array}{ll}\text { Bibliography } & 37\end{array}$

Included papers $\quad 41$

Paper I: Estimating effective boundaries of population growth in a variable environment

Paper II: Large time behavior of logistic age-structured population model

Paper III: Persistence analysis of the age-structured population model on several patches

Paper IV: Permanence of age-structured population model on several temporally variable patches 


\section{Introduction}





\section{Modeling population dynamics}

Population ecology studies changes in population size and structure and factors that may cause these changes. Starting from characteristics of individuals, such as age, stage or size, it aims to describe characteristics of a whole population, such as population density, age distribution, spatial distribution etc. Similarly, individual processes, such as birth, growth, reproduction or death, are used in description of population processes, such as population growth or variation in age distribution.

There are many different biotic and abiotic factors that, independently or combined, influence individuals in a population, which in turn, cause changes on population level. Identifying these factors and choosing the ones with the greatest impact is crucial for making a useful mathematical model of population growth. Assuming that, for example, all individuals in a population are identical with respect to age, or that a chance of reproduction or survival is always the same, is a great simplification. On the other hand, taking into account all factors that influence population dynamics would result in having too many parameters (with some of them vaguely defined and hard to measure) and possibly unsolvable mathematical model.

The challenge for mathematical modeling of population dynamics is in connecting two competing interests. On one hand, there is a demand for realistic models capable of explaining nature, predicting behavior of populations, and in the most applicable sense, that can be used for conservation and management of species. Since the ecologists would rather have qualitative instead of quantitative results, we can sacrifice precision and favor generality when we make a mathematical model of population growth. Therefore, model parameters must be measurable and meaningful from the biological perspective.. The results of the models should facilitate understanding, predicting and modifying nature. On the other hand, setting up a model and understanding it requires mathematical approach. More precisely, we need an analytical framework within which we can construct models capable of reflecting nature, as credibly as possible, and which provide, preferably, analytical solutions.

The trade-off between precision and generality will be explained in the following example. Let the number of individuals at initial time $t=0$ be $N(0)$ and suppose that the per capita birth and death rate, $b$ and $d$, respectively, are constant. The rate of change in the number of individuals can be expressed by the well-known Malthus equation:

$$
\frac{d N}{d t}=(b-d) N
$$

which leads us to the formula of exponential growth:

$$
N(t)=N(0) e^{(b-d) t} .
$$

Under the made assumptions, we obtain very precise answers to the question of population size at time $t$ : if $b>d, N(t) \rightarrow \infty$ as $t \rightarrow \infty$, if $b=d, N(t)=N(0)$ and if $b<d$, $N(t) \rightarrow 0$ as $t \rightarrow \infty$. 
However, we know for a fact that the majority of natural populations are structured by age, stage or size, that they live in temporally and spatially heterogeneous environments, and that individuals compete for resources. Although the previous model is simple and capable of predicting population explosion or extinction, it is unrealistic because it does not include any of the mentioned effects. A more realistic model of population dynamics should incorporate age (stage or size) structure, spatio-temporal heterogeneity and some sort of density-dependence. Thus, the function that represents population numbers will be dependent on two or more variables, with some of them representing population structure and time. The model is likely to have additional parameters, except the birth and death rate, depending on population structure and time.

To this end, we often use partial differential equations of the first order, or systems of partial differential equations, with different boundary and initial conditions. In these circumstances, a solution to the model, i.e., the function $N(t)$ that represents population size at time $t$, would be a function depending on the birth and death rate, the initial distribution of population into age classes and the regulating function. In some cases, model cannot be solved analytically, or it can be solved, but solution is too complicated to be of practical value.

Therefore, in order to balance the competing interests of solvability and realism, the objective of this thesis is twofold. The first objective is to formulate different population models with increasing level of realism and complexity and to prove the existence and uniqueness of a nonnegative solution for each of them. The second objective is to analyze permanence of populations under different internal and external conditions. Mathematically, we study large time behavior of a solution to the population problem. We define time-dependent upper and lower bounds of the solution, which is a new approach in solving population problem. It extends possibilities for analytical treatment of a solution, because the upper and lower bounds, as we will see, are obtained analytically and the parameters in their expressions are biologically meaningful. Analyzing the large-time behavior of the upper and lower bounds explains the large-time behavior of the function $N(t)$, gives a prediction of population growth or decline, and a proposition for practical action for conservation and management of the population.

Finally, we will evaluate if the more complex models enhance our ability to predict population growth and provide options for land management and conservation of species. The number of parameter increases with the number of ecological factors included in the model. By changing these parameters, it is possible to influence population dynamics. We are inclined to think that the models we are presenting contribute to our understanding of population dynamics and increase chance of making useful management strategies. A word of caution is, however, that the models should not be understood too literally, as the results are more qualitative than quantitative in their nature.

\subsection{Deterministic vs. stochastic models}

Ecological processes are usually stochastic. The randomness present in a ecological process originate from individuals and their characteristics, or from changes in the environ- 
ment. The former is the demographic stochasticity, which strongly affects small populations, and the latter is the environmental stochasticity, which is more important for large populations. This means that for a given initial state of population, there exists a family of trajectories and every one may occur with certain probability. Due to the fact that stochastic population models are difficult to analyze, in many cases the only option to use a model is to run a simulation.

Deterministic models, on the other hand, assume that there exists a unique trajectory for each given initial state of the population. In these models, the result can be predicted using given vital rates and initial condition. They allow deeper analysis which makes them easier to use in comparison to stochastic models.

An important property of deterministic models is that they can be seen as a limit for stochastic models under assumption that population is large. In this sense, deterministic models can be used as approximations of stochastic models. Because of this, we will consider only deterministic models in the thesis.

\subsection{Organization of the thesis}

There is more than one factor that have impact on population dynamics. In the Section 2, we will explain the ecological significance of population structure, temporal environmental variability, density-dependent factors that limit population growth and spatial heterogeneity of the environment.

The mathematical part of the thesis consist of the presentation of three fairly general models that describe population dynamics of broad groups of species. The models are presented by increasing complexity with respect to the included ecological factors.

Large time behavior of solution to an age-structured time-dependent population model is studied in Paper I. The model itself is not new, but its asymptotics analysis resolves the problem of age and time dependent vital rates and explains the influence of temporally variable environment of the large time behavior of a solution to the model. We will briefly present it in Section 3.

In Section 4 we discuss Paper II. It is a logical continuation of the work that has been done in Paper I, since it presents a model of population growth that includes densitydependence into the model analyzed in Paper I. It is assumed that a population is agestructured and lives in a variable environment. The main part of the paper is analysis of the large time behavior of a solution.

Paper III is an extension of the model from Paper II from a single habitat to several patches. Spatial structure brings new challenges for solving the boundary-initial value problem, since we have $N$ partial differential equations that define the balance law on each patch. Paper IV is mathematically more developed version of Paper III, although it is concerned with the same population problem. This can be found in Section 5.

Section 6 contains the discussion and comments about future research. 


\section{Ecological considerations}

\subsection{Why does age structure matter?}

The first population models were unstructured, i.e., variability within species was ignored. However, demographic differences between individuals in populations are undeniable, which gives rise to the concept of population structure. This means that we can divide population into classes of identical individuals with respect to their age, size, physiological state, or some other attribute. What we use as a criterion for differentiation among individuals depend on the species in question. For some, changes between individuals of different age are the most prominent. Mammals and birds fall into this category. For some others, the life cycle of consists of several recognizable morphological stages. In this case, it is reasonable to differ individuals by their stage instead of age. Typical example are insect populations. It is worth noting that there are species for which variation in size is what makes the difference. For example, the size structure is a more appropriate choice for modeling populations of microorganisms.

Unlike unstructured population models that explain dynamics of a whole population, structured population models track dynamics of the mentioned classes, and consequently they track the dynamics of the total population. Changes that we see on a population level are the result of the behavior of individuals that constitute the population.

Population response to the environmental changes often comes with a time lag, because for many effects to take place, individuals must be old enough or large enough to reproduce. Individual development and population distribution determine population dynamics. For these reasons, structured populations models can show very different behavior in comparison to unstructured models.

To illustrate the importance of age structure, we will use an example from conservation biology. Conservation biology usually deals with endangered species, which, by definition, have small populations. In 2015, the population of the northern white rhino consisted of three animals, two females and one male. All of them are considered too old to reproduce naturally, implying that the only solution for saving the subspecies from extinction is by in-vitro fertilization of a related souther white rhino surrogate. Demographic variability, i.e., age structure of the population in this case, plays a more important role than any other kind of variability (including the environmental variability). Ignoring the age of the animals in question and using unstructured population model might lead us to the false conclusion that population can survive. This puts the population structure among the most important factors for population dynamics.

Depending on the species, we can choose appropriate individual-level variable (i.e., age, stage, or size), and in addition to this, it can be discrete or continuous. This also make a distinction between age, size and stage structured models, and also a difference between discrete and continuous models. There is a long list of authors who studied age structured models, and we mention some of them: Sharpe and Lotka (1911), von Foerster (1959), McKendrick (1926), Feller (1941), Gurtin and MacCamy (1974), Iannelli (1995), Kot (2001), Webb (1985), Webb (1986). 
In what follows, we will use an age-structured, single-sex, female based model. In these types of models, a population is divided into age classes and all individuals within an age class are identical with respect to the life history traits.

\subsection{On the role of time}

The majority of natural populations live in temporally changing habitats. The changes can be climate forcing (large scale, low-frequency, positively autocorrelated changes, such as change of seasons or daily changes in temperature, humidity and light) or noise, i.e., small, stochastic, high-frequency changes. As suggested by, e.g., Stenseth et al. (2002), Bjørnstad and Grenfell (2001) and Coulson et al. (2004), neither type of changes should be ignored in modeling population dynamics, although the choice of a model and a method is still under debate.

Temporal variation affects both individuals and populations, either directly through physiology (changing metabolic processes and reproduction) or indirectly through the ecosystem (influencing prey, predators, competitors etc.). Some studies show the existence of complex interactions between different processes that shape population dynamic; see for example Steele (1985). The effects of temporal environmental change on an individual can depend on individual's age, size, stage or physiological condition. On a population level, effects of noise color depend on responsiveness of a population, as noted by Boyce and Daley (1980), Roughgarden (1979) and May (1973). For populations that respond slowly to the environmental changes, extinction risk increases for the high-frequency environmental change.

Climate forcing is easily introduced in deterministic models through time dependent vital rates, see for instance Chipot (1983), Elderkin (1985). Since the environmental changes often have recurring pattern, some authors study periodically changing environments, see for example Coleman (1979), Cook (1967), Cushing (1986a), Cushing (1986b), Tuljapurkar (1985).

\subsection{On density-dependence}

The relative importance of exogenous (environmental) factors versus endogenous (density-dependent) factors is still a matter of debate, see for example Bjørnstad and Grenfell (2001) and Coulson et al. (2004). Examples show that small periodical changes of the environment can decrease population growth, but temporal variability alone still allow a population to be unbounded. In other words, there is a lack of regulation of population growth that has been observed in natural populations. Ripa and Lundberg (1996) argue that in the case of a very small population or rather large amplitude of the environmental change, the population can be driven to extinction by the environmental variability. However, this is more a special case, than a rule. Age-structured linear models, even when they include environmental variability, are not able to explain dynamics of populations as it is observed in nature, since they allow existence of unbounded populations, as we will see later. 
Density-dependent factors (intra-specific competition and community level interactions such as predation and inter-specific competition), are, however, able to provide sufficient control of population growth and to keep population size bounded. Resources are limited in every environment and individuals in populations compete for them. In one species models, intra-specific competition is a representative of density-dependent factors. It increases with density of a population and operates more strongly on large populations providing negative feedback for population growth.

The term carrying capacity is used to define the maximal population size supported by the environment. The Verhulst model is given by:

$$
\frac{d N(t)}{d t}=r N(t)\left(1-\frac{N(t)}{K}\right), \quad N(0)=N_{0},
$$

where $r$ is the intrinsic growth rate and $K$ is the carrying capacity. A solution to the model is

$$
N(t)=\frac{K N_{0} e^{r t}}{K+N_{0}\left(e^{r t}-1\right)},
$$

which implies that the carrying capacity is a nontrivial equilibrium point, see Iannelli and Pugliese (2014). The model presupposes constant environment and unstructured population, and in turn, constant carrying capacity.

Density-dependent age-structured models are studied by, for example, Gurtin and MacCamy (1974), Webb (1986), Marcati (1983). In these cases, competition happens on population level and it is introduced in the model through density-dependent birth and death rates.

In temporally variable environments it would be reasonable to assume that the carrying capacity is changing with respect to time. For age-structured populations, biologically explainable is a situation when different age classes compete for different resources, or when the strength of competition between individuals depends on their age classes. This can be a motivation for using age-dependent carrying capacity.

\subsection{Importance of spatial heterogeneity}

Apart from being temporally variable, a natural environment is often spatially heterogeneous. Human activities are changing landscape and cause reduction and fragmentation of habitats. This, in turn, contributes to destabilization of populations and loss of biodiversity. Some authors argue that better understanding of spatio-temporal dynamics of populations would lead to the better management of land use and to the more successful conservation of species. Thus, the final challenge in modeling population dynamics is including space in population models. See, for example, Kareiva and Wennergren (1995), Wennergren et al. (1995), Bjørnstad and Grenfell (2001).

Spatial heterogeneity can be treated by continuous-space models or discrete-space models, see Vance (1984). The continuous-space models assume that a population lives in a single heterogeneous habitat and movement of individuals within the habitat is defined by diffusion. Finding a solution to these models requires advanced mathematical 
techniques and can be very challenging. The discrete-space models presuppose that the population occupies several habitats and the movement of individuals is described as dispersal between habitats. Modelers have options to assume that all habitats are identical (which leads to metapopulation models) or to consider that each patch is unique and, as such, gives rise to the local subpopulation dynamics (which leads to the sourcesink dynamics). In both cases, the effects of dispersion on persistence and extinction of population are considerate and they were studied by various authors, for example Allen (1983) and Hastings (1993).

We will briefly explain the source-sink dynamics, because in the spatial models we are using this approach. If a species lives on several different patches, it is of interest to estimate the contribution of each patch to the persistence of the whole population. Individual patch contribution (or quality) depends on demography of the local subpopulation. A source is a high quality patch in terms of the high birth rate and survival. A subpopulation on the source is persistent (or even growing) without immigration. Contrary to this, a sink is a low quality patch, on which the subpopulation is declining and eventually goes to extinction without influx of immigrants. Sources and sinks are connected by dispersal of individuals even when they are not physically close. Sources can be seen as exporters of individuals, while subpopulations on sink patches depend on individuals coming from sources for survival.

The source-sink dynamics described above is not a novelty, see Allen (1983), Pulliam (1988), Dias (1996). The influence of dispersal on survival of populations on sinks has already been investigated. It is understood that dispersion from a source to a sink can save a population on the sink from extinction. Moreover, a single source patch can support several sinks, which implies that extinction of a population on a source can cause a collapse of the whole system. This is not always the case, since some research indicates that survival of population is possible even if it occupies only sink patches, see Jansen and Yoshimura (1998).

The effect of dispersal on permanence of an unstructured population in a variable patchy environment has been studied by various authors, see for example Chi and Chen (1998), Chi and Chen (2001), Takeuchi (1986a), Takeuchi (1986b). In the case of agestructured models, a two or three-patch system involving a species that has two ageclasses has been studied, So et al. (2001), Terry (2011), Weng et al. (2010). It is our interest to study an age-structured population that inhabits $N$ temporally varying patches. By combining age-structure and source-sink dynamics, we will demonstrate that population permanence on all patches is possible for a particular dispersion pattern. We will also show that in some cases, a population can survive even if all patches are sinks. This leads us to migratory birds because their habitats may be considered as sinks. The migration is then an extreme example of a population dynamics influenced by spatiotemporal variability. 


\section{On linear age-structured population model in a variable environment}

\subsection{Constant environment}

The classical age-structured Lotka-McKendrick-von Foerster population model considers a population divided into age classes consisting of identical individuals with respect to their vital rates. In this case, the population density function $N(t)$ is not sufficient to fully describe population dynamics (as it was, for example, in the Malthus or in the Verhulst population models). To encompass population structure and its implications for population dynamics, the function $n(a, t)$ which represents density of age class $a$ at time $t$ is used.

The death and aging processes satisfy the linear partial differential equation

$$
\frac{\partial n(a, t)}{\partial t}+\frac{\partial n(a, t)}{\partial a}=-\mu(a) n(a, t), \quad a, t>0,
$$

where $\mu(a)$ is the age-dependent death rate. The birth process is defined by the so-called renewal equation

$$
n(0, t)=\int_{0}^{\infty} m(a) n(a, t) d a, \quad t>0,
$$

where a nonnegative function $m(a)$ is the age-dependent birth rate, and the initial condition is

$$
n(a, 0)=f(a), \quad a>0,
$$

where a nonnegative function $f(a)$ is the initial distribution of population in age classes. Additionally, a number of assumptions are used to make biologically reasonable model.

The total population $N(t)$ at time $t$ is defined by

$$
N(t)=\int_{0}^{\infty} n(a, t) d a .
$$

Since this number should be finite, it is reasonable to assume that $n(\cdot, t) \in L^{1}\left(\mathbb{R}^{+}\right)$for every $t \geq 0$. In order to get a global solution, $n \in L^{\infty}\left([0, T], L^{1}\left(\mathbb{R}^{+}\right)\right)$, for arbitrary $T>0$.

The problem (3.1)-(3.3) is a linear hyperbolic partial differential equation with boundary and initial conditions. It can be solved by the method of characteristics. Using the notation

$$
\rho(t)=n(0, t),
$$

and integrating along characteristics, we obtain an expression for population densities $n(a, t)$ for $a, t \geq 0$. Namely, the following holds:

$$
\begin{aligned}
\rho(t)= & \int_{0}^{t} m(a) e^{-\int_{0}^{a} \mu(v) d v} \rho(t-a) d a \\
& +\int_{t}^{\infty} m(a) e^{-\int_{a-t}^{a} \mu(v) d v} f(a-t) d a
\end{aligned}
$$


for $t>0$. For $a>0$, value of the function $n(a, t)$ is given by

$$
n(a, t)= \begin{cases}\rho(t-a) e^{-\int_{0}^{a} \mu(v) d v}, & 0<a<t, \\ f(a-t) e^{-\int_{a-t}^{a} \mu(v) d v}, & a \geq t .\end{cases}
$$

Various authors have proved existence and uniqueness of a solution to problem (3.1)(3.3). Details and proofs can be found in, e.g., Sharpe and Lotka (1911), Kot (2001), Webb (1985).

In order to study the asymptotic behavior of a solution to equation (3.4), suppose that the solution is of the form $e^{\lambda t}$. This leads us to the following integral equation:

$$
\int_{0}^{\infty} m(a) e^{-\lambda a-\int_{0}^{a} \mu(v) d v} d a=1
$$

Equation (3.6) is known as the Euler-Lotka characteristic equation. The largest root of equation (3.6) is real. According to Iannelli and Pugliese (2014), asymptotics of the function $\rho(t)$ satisfies

$$
\rho(t)=c e^{\lambda t}(1+\Omega(t)), \quad \text { where } \quad \lim _{t \rightarrow \infty} \Omega(t)=0 .
$$

Therefore, for $\lambda>0, \rho(t) \rightarrow \infty$, for $\lambda=0, \rho(t) \rightarrow c$, and for $\lambda<0, \rho(t) \rightarrow 0$ as $t \rightarrow \infty$. In other words, extinction or explosion of a population depends on the sign of $\lambda$.

The net reproductive rate ${ }^{1}$, denoted by $R_{0}$, represents the average number of offspring per individual during the whole lifetime. It is defined by

$$
R_{0}=\int_{0}^{\infty} m(a) e^{-\int_{0}^{a} \mu(v) d v} d a,
$$

and it can be used for predicting population growth. Namely, the well-known result connects $R_{0}$ and solution $\lambda$ to the characteristic equation (3.6):

$$
\left.\left.R_{0}>1 \text { (resp. } R_{0}<1\right) \Leftrightarrow \lambda>0 \text { (resp. } \lambda<0\right) \text {. }
$$

For details, see Iannelli (1995). In other words, the following holds:

$$
\begin{aligned}
& R_{0}>1 \Rightarrow \rho(t) \rightarrow \infty \text { as } t \rightarrow \infty, \\
& R_{0}=1 \Rightarrow \rho(t) \rightarrow c \text { as } t \rightarrow \infty, \\
& R_{0}<1 \Rightarrow \rho(t) \rightarrow 0 \text { as } t \rightarrow \infty .
\end{aligned}
$$

Combining the definition of $N(t)$ and (3.5), one can show that similar result holds for the large-time behavior of the total population.

\footnotetext{
${ }^{1}$ Worth mentioning is that different authors use various terms to denote $R_{0}$, with basic reproduction ratio and reproduction number being only some of them.
} 


\subsection{Temporally varying environment}

Chipot (1983) and Elderkin (1985) extended the linear age-structured model (3.1)-(3.3) by assuming that populations inhabits temporally changing environments. The environmental changes are represented by the time-dependent vital rates, which leads to the following model:

$$
\begin{aligned}
\frac{\partial n(a, t)}{\partial t}+\frac{\partial n(a, t)}{\partial a} & =-\mu(a, t) n(a, t), \quad a, t>0, \\
n(0, t) & =\int_{0}^{\infty} m(a, t) n(a, t) d a, \quad t>0, \\
n(a, 0) & =f(a), \quad a>0,
\end{aligned}
$$

where $m(a, t)$ and $\mu(a, t)$ are the birth and death rate, respectively, and $f(a)$ is the initial distribution of population. The functions $m$ and $f$ satisfy: $m(a, t) \in L^{\infty}\left([0, T] \times \mathbb{R}^{+}\right)$and $f \in L^{1}\left(\mathbb{R}^{+}\right)$.

\subsection{Asymptotics of the linear age-structured time-dependent model}

The main objective of Paper I was to study the asymptotic behavior of an age-structured population in temporally variable environment. The model we used in Paper I is given by the same equations as model (3.9), with the following assumptions: (1) the constant $A_{\mu}$ denotes an upper bound for the maximal length of life of individuals in population. The constant $A_{m}$ is the upper bound of fertility period and $A_{m} \leq A_{\mu}$. (2) the birth rate $m$, the death rate $\mu$, and the initial distribution $f$ of a population into age classes are measurable and nonnegative functions with the following properties:

(i) $m(a, t)$ is bounded for $a, t \geq 0$,

$m(a, t)=0$ for $a>A_{m}$ and $t \geq 0$,

$m(a, t) \geq \delta_{1}>0$ for $a_{1}<a<a_{2}$, where $0<a_{1}<a_{2}<A_{m}$ and $t \geq 0$,

(ii) $0<c_{\mu} \leq \mu(a, t) \leq C_{\mu}<\infty$ for $a \leq a_{2}$ and $t \geq 0$,

$\int_{A}^{A+A_{\mu}} \mu(a, t) d a=\infty$ for $t \geq 0$ and $A \geq 0$,

(iii) $f$ is bounded,

$f(a) \geq \delta_{2}>0$ for $a \in\left(b_{1}, b_{2}\right), b_{2}<a_{2}$,

$f(a)=0$ for $a>A_{\mu}$.

After integration along characteristics, we obtain the expression for a solution $n(a, t)$ to the problem (3.9):

$$
n(a, t)= \begin{cases}\rho(a-t) e^{-\int_{0}^{a} \mu(\nu, v+t-a) d v}, & 0<a<t, \\ f(a-t) e^{-\int_{a-t}^{a} \mu(\nu, v+t-a) d v}, & a \geq t\end{cases}
$$


Similarly to the equation (3.4), here we have the integral equation

$$
\rho(t)=K \rho(t)+F(t), \quad t \geq 0,
$$

where

$$
K \rho(t)=\int_{0}^{t} m(a, t) e^{-\int_{0}^{a} \mu(v, v+t-a) d v} \rho(t-a) d a, \quad t \geq 0,
$$

and

$$
F(t)=\int_{t}^{\infty} m(a, t) e^{-\int_{a-t}^{a} \mu(v, v+a-t) d v} f(a-t) d a, \quad t \geq 0 .
$$

Given a positive real number $\Lambda$, let $L_{\Lambda}^{\infty}(0, \infty)$ denote a space of measurable functions $u$ on $[0, \infty)$ such that $|u(t)|=O\left(e^{\Lambda t}\right)$ for $t \geq 0$. The norm on $L_{\Lambda}^{\infty}(0, \infty)$ is defined by

$$
\|u\|_{\Lambda}=\operatorname{esssup}_{t>0}|u(t)| e^{-\Lambda t}
$$

and $L_{\Lambda}^{\infty}(0, \infty)$ is Banach space for every real $\Lambda$.

One can show that the operator $K$ is a contraction for sufficiently large $\Lambda$. Using a fixed point argument, we prove that equation (3.11) has a unique solution $\rho \in L_{\Lambda}^{\infty}(0, \infty)$. This, in turn, guarantees existence and uniqueness of a solution $n(a, t)$ to the problem (3.9).

\subsubsection{General upper and lower bounds}

The Euler-Lotka characteristic equation (3.6) led to an explanation of the large-time behavior of a solution to the model (3.1). In analogy to this, we are looking for a characteristic equation that would allow us to describe asymptotics of a solution to the model (3.9).

To this end, we assume that a solution to equation (3.11) is of the form $e^{\int_{0}^{t} \sigma(\tau) d \tau}$, for some $\sigma \in L^{\infty}[0, \infty)$. Plugging it in (3.11) and using that $F(t)=0$ for $t \geq M$, where $M>A_{m}$, we obtain the characteristic equation for the time-dependent model:

$$
\int_{0}^{\infty} m(a, t) e^{-\int_{0}^{a} \mu(\nu, v+t-a) d v-\int_{t-a}^{t} \sigma(\tau) d \tau} d a=1 \quad \text { for } t>M
$$

where $M>A_{m}$.

If $\sigma(t)$ is a solution to the equation (3.14), then both the number of newborns, $\rho(t)$, and the total population, $N(t)$, can be bounded from above and below by some functions that depend on $\sigma(t)$. Namely, we prove that

$$
C_{1} e^{\int_{0}^{t} \sigma(\tau) d \tau} \leq \rho(t) \leq D_{1} e^{\int_{0}^{t} \sigma(\tau) d \tau}
$$

and

$$
C_{2} e^{\int_{0}^{t} \sigma(\tau) d \tau} \leq N(t) \leq D_{2} e^{\int_{0}^{t} \sigma(\tau) d \tau},
$$

for sufficiently large $t$ and some positive constants $C_{1}, C_{2}, D_{1}$ and $D_{2}$.

If instead of equality in (3.14) we have inequalities $\leq$ (or $\geq$ ), then the right hand side (or the left hand side) inequalities in (3.15) and (3.16) still hold. 
Finding a solution $\sigma(t)$ to the equation (3.14) analytically can be difficult, if not impossible. Thus, when it comes to predicting population growth, the previous general result is of little practical use. In order to investigate the asymptotics of the function $\rho(t)$ that solves (3.11), we use its upper and lower bounds. if

Recall that a nonnegative function $\rho^{+} \in L_{\Lambda}^{\infty}(0, \infty)$ is an upper solution to equation (3.11)

$$
\rho^{+}(t) \geq K \rho^{+}(t)+F(t) \text { for } t \geq 0 .
$$

Similarly, a nonnegative function $\rho^{-} \in L_{\Lambda}^{\infty}(0, \infty)$ is a lower solution to equation (3.11) if

$$
\rho^{-}(t) \leq K \rho^{-}(t)+F(t) \text { for } t \geq 0 .
$$

The importance of this definition is that the upper and lower solutions to equation (3.11) give upper and lower bounds for the function $\rho(t)$ for $t \geq 0$. Namely, the following holds:

$$
\rho^{-}(t) \leq \rho(t) \leq \rho^{+}(t) \text { for } t \geq 0 .
$$

For the details, see Section 7.4 in Zeidler (1986).

In the next sections, we will use time-independent models and periodically changing models to formulate upper and lower solutions to equation (3.14) and explain asymptotics of the function $\rho(t)$.

\subsubsection{Upper and lower bounds through time-independent model}

From the ecological point of view, it is more important to know population dynamics than to know its exact size. Thus, having upper and lower bounds of the function $\rho(t)$ instead of having the exact value of $\rho(t)$ is a good trade-off between solvability of a problem and precision.

We formulate two auxiliary time-independent models that will provide upper and lower bounds for the original problem (3.9). A good environment provides high birth rate and survival, while for a bad environment the opposite is true. Taking the maximal value of the birth rate and the minimal value of the death rate with respect to time, enables us to define the best case scenario. Similarly, the minimal birth rate and the maximal death rate define the worst case scenario. The best and the worst cases represent constant environments, which implies time-independent vital rates and the following characteristic equations:

$$
\int_{0}^{\infty} m_{+}(a) e^{-k_{+} a-\int_{0}^{a} \mu_{-}(v) d v} d a=1
$$

and

$$
\int_{0}^{\infty} m_{-}(a) e^{-k_{-} a-\int_{0}^{a} \mu_{+}(v) d v} d a=1 .
$$

Using the solutions $k_{ \pm}$to define the function $\sigma$, we obtain the following upper and lower bounds for the functions $\rho(t)$ and $N(t)$ :

$$
C_{1} e^{k_{-} t} \leq \rho(t) \leq D_{1} e^{k_{+} t} \text { for large } t,
$$


and

$$
C_{2} e^{k_{-} t} \leq N(t) \leq D_{2} e^{k_{+} t} \quad \text { for large } t,
$$

where $C_{i}, D_{i}>0, i=1,2$.

This makes solving the characteristic equation (3.14) redundant, because we can study the large time behavior of a population by examining properties of mentioned bounds.

\subsubsection{Periodical variation of environment}

Environments are often changing periodically. The changes include, for example, daily changes in temperature, humidity and light, change of seasons, predation or availability of food, etc. We assume that the birth rate is affected by the environmental changes and the death rate depends only on age. Therefore, the birth rate is of the form

$$
m(a, t)=m(a)(1+\varepsilon \cos A(t-\gamma)), \quad a, t \geq 0,
$$

where $A>0, \gamma \geq 0$ and $\varepsilon>0$ is a small number. For simplicity, we assume that the death rate is time-independent, i.e., $\mu(a, t) \equiv \mu(a)$.

In this case, we obtain explicit upper and lower bounds for the functions $\rho(t)$ and $N(t)$. Namely, let $k_{0}$ be a solution to equation

$$
\int_{0}^{\infty} Q(a) e^{-k_{0} a} d a=1
$$

where $Q(a)=m(a) e^{-\int_{0}^{a} \mu(v) d v}$ and let $k_{2}$ be equal to

$$
k_{2}(A)=\frac{1}{2 T}\left(\frac{1-I_{c}(A)}{I_{s}^{2}(A)+\left(1-I_{c}(A)\right)^{2}}-1\right)
$$

where

$$
I_{c}(A)=\int_{0}^{\infty} Q(a) e^{-k_{0} a} \cos A a d a \quad \text { and } \quad I_{s}(A)=\int_{0}^{\infty} Q(a) e^{-k_{0} a} \sin \text { Aada. }
$$

For sufficiently large $t$, we have that

$$
C_{1} e^{\left(k_{0}+\epsilon^{2} k_{2}-\alpha \epsilon^{3}\right) t} \leq \rho(t) \leq D_{1} e^{\left(k_{0}+\epsilon^{2} k_{2}+\alpha \epsilon^{3}\right) t},
$$

and

$$
C_{2} e^{\left(k_{0}+\epsilon^{2} k_{2}-\alpha \epsilon^{3}\right) t} \leq N(t) \leq D_{2} e^{\left(k_{0}+\epsilon^{2} k_{2}+\alpha \epsilon^{3}\right) t},
$$

where $C_{i}, D_{i}, i=1,2$ and $\alpha$ are positive constants and $\varepsilon>0$ is a small number.

Since $\varepsilon$ is a small number, the asymptotic behavior of the number of newborns $\rho(t)$ and the total population $N(t)$ is determined by the parameter $k_{0}$. For $k_{0}>0$, population is growing, while for $k_{0}<0$ it is declining. In the special case when $k_{0}=0$, the large time behavior of population is entirely determined by the sign of $k_{2}(A)$, which is, according to (3.18), a function depending on frequency $A$. 


\subsubsection{Conclusions}

In the discrete population model presented by Tuljapurkar (1985), population growth is governed by the average vital rates. Our analysis provides the same conclusion, since the parameter $k_{0}$ depends on the vital rates and life history, as a solution to equation (3.17). The assumption that $\varepsilon$ is a small number contributes to the fact that $k_{0}$ has dominant role in determining asymptotics of a solution, except in case when $k_{0}=0$. For $k_{0}=0$, the large-time behavior of the population depends on the sign of the parameter $k_{2}$.

We used four different life histories to examine $k_{2}$ as a function of frequency of oscillation. Since $k_{2}$ changes sign, as we can see in Figure 3.3.4, its effect on population growth differ depending on the frequency.
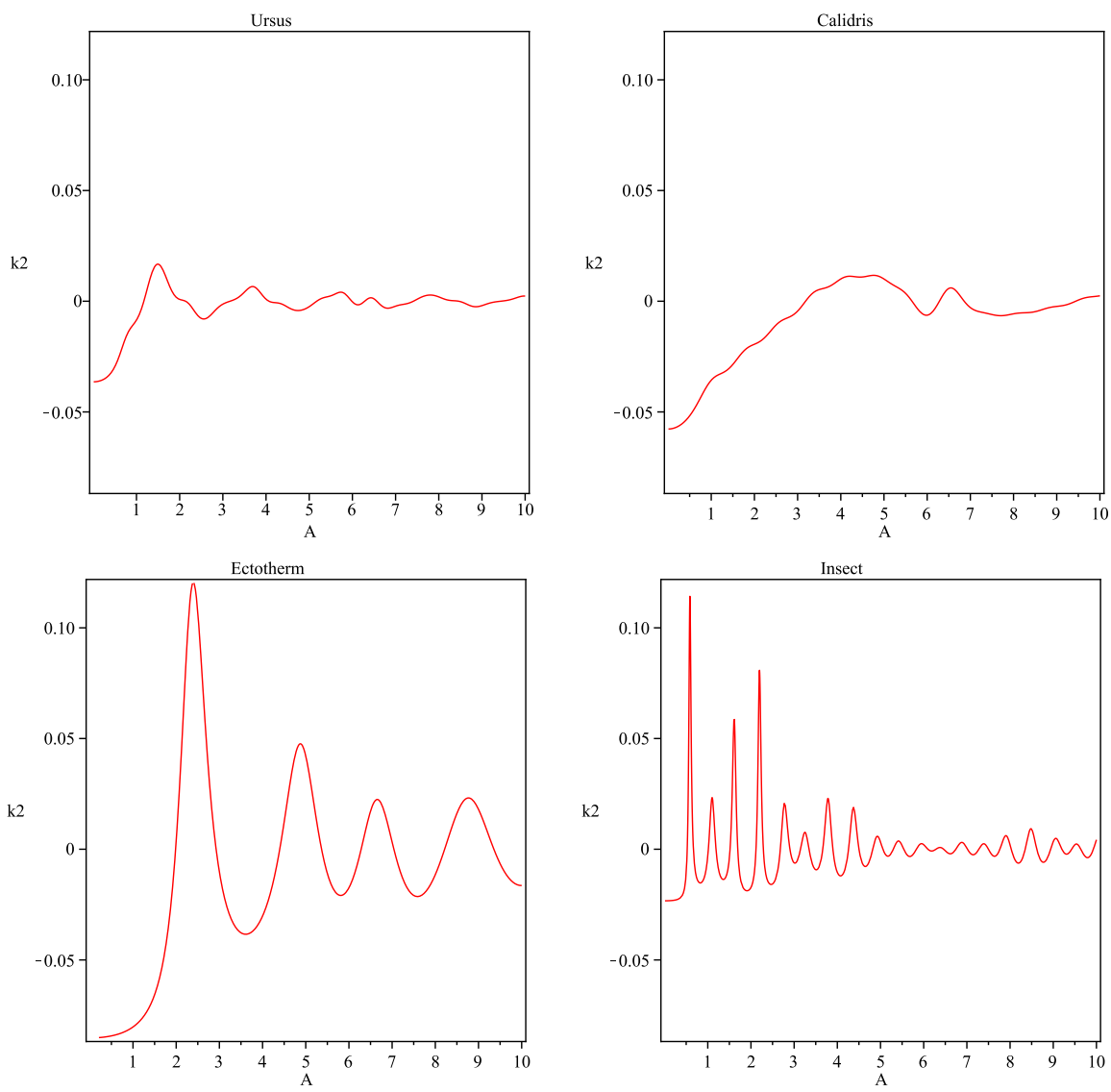

Figure 1: $k_{2}$ as a function of $A$

Common for all life histories it that a low-frequency variation has detrimental effect on population growth. For other frequencies, there is no common response for all 
species. According to Tuljapurkar, growth rate is increased by oscillations with periods near generation time and decreased by oscillations with much shorter or much longer periods. Unlike him, we show that if the period of oscillation is comparable to the generation time, one needs to consider life history as well. This is motivated by the fact that different species have different responses to changes in the environment. This observation imply that there is a deeper connection between age-structure and temporal variability that should be investigated. 


\section{Logistic age-structured population model in a variable environment}

\subsection{Modeling density-dependence}

Including density-dependent factors into age-structured models is not a novelty. For example, the model by Gurtin and MacCamy (1974) presupposes that the vital rates depend on age and on the population density. Chipot (1983) and Elderkin (1985) extended the nonlinear model so that it includes time-dependent vital rates. Existence of a unique solution has been proven in these cases.

On the other hand, density-dependent growth is often connected to the concept of the carrying capacity, which comes with its own vagueness, Price (1999). In the classical Verhulst model, the environment is constant and it imposes a limit to the maximal sustainable population size. This limit is usually identified with the carrying capacity. A population stabilizes when it reaches the carrying capacity or fluctuates around it.

Traditionally, it has been assumed that food is the limiting factor for population growth. For numerous natural populations this is not the case. Depending on a population, the limiting factor can be anything from available water, nesting places, places to hide from predators, material for building nests, exposure to a disease etc. This gives rise to several observations. First, in age-structured populations, individuals from different age classes can, but do not have to compete for a same resource. Even if we consider food as the limiting factor of the population growth, we notice that insect of different stages do not feed on the same resource. Therefore, no competition on the population level is present and the function that defines competition should reflect this. Second, in temporally varying environments, availability of the resource or exposure to predators and disease can change with time. Hence, the limiting factor should be represented by a time-dependent function.

\subsection{Age-structured model with logistic term}

Starting from the age-structured model (3.9) and assuming that competition occurs only within individuals in the same age class and contributes only to mortality, we get the following model of population growth:

$$
\begin{aligned}
\frac{\partial n(a, t)}{\partial t}+\frac{\partial n(a, t)}{\partial a} & =-\mu(a, t) n(a, t)\left(1+\frac{n(a, t)}{L(a, t)}\right), \quad a, t>0, \\
n(0, t) & =\int_{0}^{\infty} m(a, t) n(a, t) d a, \quad t>0, \\
n(a, 0) & =f(a), \quad a>0 .
\end{aligned}
$$

As before, the functions $m(a, t)$ and $\mu(a, t)$ are per capita birth and death rate, respectively, and $f(a)$ is the initial distribution of population into age classes. The function $L(a, t)$ will be called the regulating function, and its purpose will be explained shortly. 
In the classical Verhulst model (2.1), the logistic term represents density-dependent mortality. Following this line of thought, we formulate the logistic term $\frac{\mu(a, t) n^{2}(a, t)}{L(a, t)}$ to express the density-dependent increase in mortality. The regulating function $L(a, t)$ represents limitations imposed on individuals by the environment (or available resource per capita). For example, if $L(a, t)=n(a, t)$ for some $a, t \geq 0$, then the mortality of age class doubles in comparison to mortality in the linear density-independent population model. If the environment is poor, density-dependent mortality increases, i.e., $L(a, t) \rightarrow$ 0 implies $\frac{\mu(a, t) n^{2}(a, t)}{L(a, t)} \rightarrow \infty$. Conversely, in rich environment, density-dependent mortality decreases, i.e., $L(a, t) \rightarrow \infty$ implies $\frac{\mu(a, t) n^{2}(a, t)}{L(a, t)} \rightarrow 0$.

\subsubsection{Existence and uniqueness of a solution}

In order to have biologically meaningful model, we introduce several assumptions. The constant $A_{\mu}$ is the maximal length of life of individuals in population and the interval of fertility of individuals is $\left(a_{m}, A_{m}\right)$. Notice that $\left(a_{m}, A_{m}\right) \subset\left[0, A_{\mu}\right]$. The vital rates, initial distribution and regulating function are measurable, nonnegative functions such that:

(i) $m$ is bounded for all $a, t \geq 0$,

$m(a, t) \geq \delta>0$ if $a \in\left(a_{1}, a_{2}\right)$, where $0<a_{m}<a_{1}<a_{2}<A_{m}$,

$m(a, t)=0$ if $a \notin\left(a_{m}, A_{m}\right)$.

(ii) $0<c_{\mu} \leq \mu(a, t) \leq C_{\mu}<\infty$ for $a \in\left(0, A_{m}\right)$,

$\int_{T}^{A_{\mu}+T} \mu(a, t) d a=\infty$ for $T \geq 0$.

(iii) $f$ is bounded for $a \geq 0$,

$f(a) \geq \delta_{1}>0$ for $a \in\left(b_{1}, b_{2}\right)$, where $b_{2}<a_{2}$

$f(a)=0$ for $a>A_{\mu}$.

(iv) $0<L_{1} \leq L(a, t) \leq L_{2}<\infty$ for all $a, t \geq 0$.

Integrating along characteristics, we show that the function $n(a, t)$ that solves the problem (4.1) have the following representation:

$$
n(a, t)= \begin{cases}\frac{\rho(t-a) e^{-\int_{0}^{a} \mu(v, v+t-a) d v}}{1+\rho(t-a) \pi(a, t)}, & a<t \\ \frac{f(a-t) e^{-\int_{a-t}^{a} \mu(\nu, v+t-a) d v}}{1+f(a-t) \phi(a, t)}, & a \geq t,\end{cases}
$$

where

$$
\begin{aligned}
& \pi(a, t)=\int_{0}^{a} \frac{\mu(\nu, v+t-a) e^{-\int_{0}^{v} \mu(s, s+t-a) d s}}{L(\nu, v+t-a)} d v, \quad t>a, \\
& \phi(a, t)=\int_{a-t}^{a} \frac{\mu(\nu, v+t-a) e^{-\int_{a-t}^{v} \mu(s, s+t-v) d s}}{L(\nu, v+t-a)} d v, \quad a>t, \\
& \psi(a, t)=m(a, t) e^{-\int_{a-t}^{a} \mu(\nu, v+t-a) d v}, \quad a>t .
\end{aligned}
$$


We introduce the notation $\rho(t)=n(0, t)$ and have that

$$
\rho(t)=\int_{0}^{t} \frac{Q(a, t) \rho(t-a)}{1+\rho(t-a) \pi(a, t)} d a+\int_{t}^{\infty} \frac{\psi(a, t) f(a-t)}{1+f(a-t) \phi(a, t)} d a,
$$

where

$$
Q(a, t)=m(a, t) e^{-\int_{0}^{a} \mu(\nu, v+t-a) d v}, \quad t>a .
$$

In order to show that the problem (4.1) has a unique solution, we prove that solution to (4.4) is unique. We begin by writing equation (4.4) in the operator form. The first term in (4.4) defines a nonlinear, monotone and Lipschitz continuous operator on $L^{\infty}(0, \infty)$. Using these properties, one can prove existence and uniqueness of a solution to (4.4).

\subsubsection{Asymptotics in a constant environment}

In case of a constant environment, the vital rates and the regulating function are timeindependent and the characteristic equation is of the form

$$
\int_{0}^{\infty} \frac{m(a) e^{-\int_{0}^{a} \mu(\nu) d v}}{1+\rho^{*}\left(1-\frac{1}{L} e^{-\int_{0}^{a} \mu(\nu) d \nu}\right)} d a=1 .
$$

Recall that the net reproductive rate $R_{0}$ is defined by

$$
R_{0}=\int_{0}^{\infty} m(a) e^{-\int_{0}^{a} \mu(v) d v} d a .
$$

If $R_{0}>1$, there exists a unique solution $\rho^{*}>0$ to equation (4.5). We can show that for large $t$ the following holds:

$$
\rho(t)=\left\{\begin{array}{ccc}
\rho^{*}+O\left(e^{-\alpha t}\right) & \text { if } \quad R_{0}>1, \\
O\left(\frac{1}{1+t}\right) & \text { if } \quad R_{0}=1, \\
O\left(e^{-\alpha t}\right) & \text { if } & R_{0}<1 .
\end{array}\right.
$$

Combining definition of the total population with estimates of the function $\rho(t)$, we arrive at the expression for the function $N(t)$ for large $t$ :

$$
N(t)=\left\{\begin{array}{ccc}
C \rho^{*}+O\left(e^{-\alpha t}\right) & \text { if } \quad R_{0}>1, \\
O\left(\frac{1}{1+t}\right) & \text { if } & R_{0}=1, \\
O\left(e^{-\alpha t}\right) & \text { if } & R_{0}<1
\end{array}\right.
$$

where $C=\int_{0}^{A_{\mu}} \frac{e^{-\int_{0}^{a} \mu(v) d v}}{1+\frac{\rho^{*}}{L}\left(1-e^{-\int_{0}^{a} \mu(v) d v}\right)} d a$ and $\alpha$ is a positive constant.

We have already motivated the need for a function that would represent the strength of competition between individuals. We have also pointed out that the carrying capacity, as we know it, might not fit in the model. These were the reasons for introducing the regulating function $L$, which acts as a measure of limitation of the resources. Results from 
this section show that the number of newborns and the total population converge to $\rho^{*}$ and $C \rho^{*}$, respectively. The constants $\rho^{*}$ and $C$ are finite and depend on the regulating function and the life history. However, in general, they are not equal to the regulating function $L$. This is in sharp contrast to the Verhulst model, but it underlines importance of having population model that combines age-structure and density-dependence.

\subsubsection{Asymptotics in a variable environment}

The asymptotics in a variable environment is assessed using auxiliary time-independent models, as it is done in Section 3.3.2 for the linear model. By taking the supremum and infimum of the vital rates and regulating function for large time, we get their timeindependent counterparts. They are used in formulation of the time-independent best and the worst case problems. Hence, solutions to these problems are possible to obtain and analyze, as we have seen in the previous section. The best case scenario defines an upper bound to the original problem, while the worst case scenario gives a lower bound. Thus, if $\rho(t)$ is the solution to the original time-dependent problem and $\rho_{-}(t)$ and $\rho_{+}(t)$ are the worst and the best case solutions, it follows that

$$
\rho_{-}(t) \leq \rho(t) \leq \rho_{+}(t) .
$$

If $R_{0}^{+}$and $R_{0}^{-}$are the net reproductive rates in the best and in the worst case and $\rho_{+}^{*}$ and $\rho_{-}^{*}$ are solutions to the corresponding characteristic equations, we obtain two-side estimates for the number of newborns and for the total population as follows:

$$
\begin{gathered}
\rho_{-}^{*}+O\left(e^{-\alpha_{-} t}\right) \leq \rho(t) \leq \rho_{+}^{*}+O\left(e^{-\alpha_{+} t}\right) \text { if } R_{0}^{-}>1, \\
\rho(t) \leq O\left(e^{-\alpha t}\right) \text { if } R_{0}^{+} \leq 1,
\end{gathered}
$$

and

$$
\begin{gathered}
C_{-} \rho_{-}^{*}+O\left(e^{-\alpha_{-} t}\right) \leq N(t) \leq C_{+} \rho_{+}^{*}+O\left(e^{-\alpha_{+} t}\right) \text { if } R_{0}^{-}>1, \\
N(t)=O\left(e^{-\alpha t}\right) \quad \text { if } \quad R_{0}^{+} \leq 1
\end{gathered}
$$

for certain positive constants $C_{ \pm}$and $\alpha_{ \pm}$.

Thus, regardless of the pattern of environmental change, the total population lies within the boundaries predicted by the best and worst case solutions. The extinction risk can be predicted in accordance to the net reproductive rates in the two extreme cases.

\subsubsection{Periodically changing environment}

The majority of natural environments are changing periodically. Climate forcing affects individuals by changing their vital rates. For simplicity, suppose that environmental 
variability has influence only on the birth rate, leaving the death rate and the regulating function unaffected, i.e.,

$$
\begin{aligned}
m(a, t) & =m_{0}(a)+\varepsilon \cos (A t) m_{1}(a), \quad a, t>0, \\
\mu(a, t) & =\mu(a), \\
L(a, t) & \equiv L, \quad L>0,
\end{aligned}
$$

where $m_{0}$ and $m_{1}$ satisfy assumption (i) and $A, \varepsilon>0$. If we additionally suppose that the average net reproductive rate is strictly larger than one, then the number of newborns can be calculated by:

$$
\begin{aligned}
\rho(t)= & \rho_{0}^{*}+\varepsilon\left(c_{1} \cos A t+d_{1} \sin A t\right) \\
& +\varepsilon^{2}\left(k_{2}+c_{2} \cos 2 A t+d_{2} \sin 2 A t\right)+O\left(\varepsilon^{3}\right),
\end{aligned}
$$

where $\rho_{0}^{*}$ is a solution to the characteristic equation

$$
\int_{0}^{\infty} \frac{m_{0}(a) e^{-\int_{0}^{a} \mu(\nu) d v}}{1+\rho_{0}^{*}\left(1-e^{-\int_{0}^{a} \mu(v) d v}\right)} d a,
$$

and $c_{1}, d_{1}, c_{2}, d_{2}$ and $k_{2}$ are certain parameters depending on the vital rates and period of oscillation $A$. Moreover, the average number of newborns can be found by

$$
\rho_{a v}=\rho_{0}^{*}+\varepsilon^{2} k_{2}+O\left(\varepsilon^{3}\right)
$$

and the average total population is

$$
N_{a \nu}=C \rho_{0}^{*}+\varepsilon^{2} C k_{2}+O\left(\varepsilon^{3}\right)
$$

where $C$ is a positive constant depending on the vital rates and the maximal length of life.

This implies that oscillations in the birth rate, caused by the changes in environment, have effects on the number of newborns and on the total population and on their average values. Depending on the sign of the parameter $k_{2}$, effect of the environmental change can be beneficial or detrimental for population growth.

\subsubsection{Conclusions}

Using life tables for four different species, we plotted $k_{2}$ as a function of frequency of oscillation $A$, see Figure 4.2.5. According to this, all oscillations, except the ones with very low-frequencies, are detrimental for population growth, because they give negative $k_{2}$.

Ripa and Lundberg (1996) claim that temporal variability can be a cause of extinction in case of very small populations or when the amplitude of oscillation is sufficiently large. By (4.6) and (4.7), we conclude that this can happen if $\rho_{0}^{*}<\varepsilon^{2}\left|k_{2}\right|$. The maximal absolute value of $k_{2}$ depends on the life history, according to the Figure 4.2.5, which 
means that some species are more sensitive to environmental fluctuations that the others. This is somewhat similar to Roughgarden (1979). She argues that fluctuations of the environment cause discrepancy between population size and carrying capacity and that relation between population ability to track fluctuating resources and predictability of the environment can be found.

Roughgarden (1975), May (1973) and Boyce and Daley (1980) claim that environmental variation keeps population numbers below its carrying capacity. We come to the similar conclusion, since almost every environmental variation yields negative $k_{2}$.
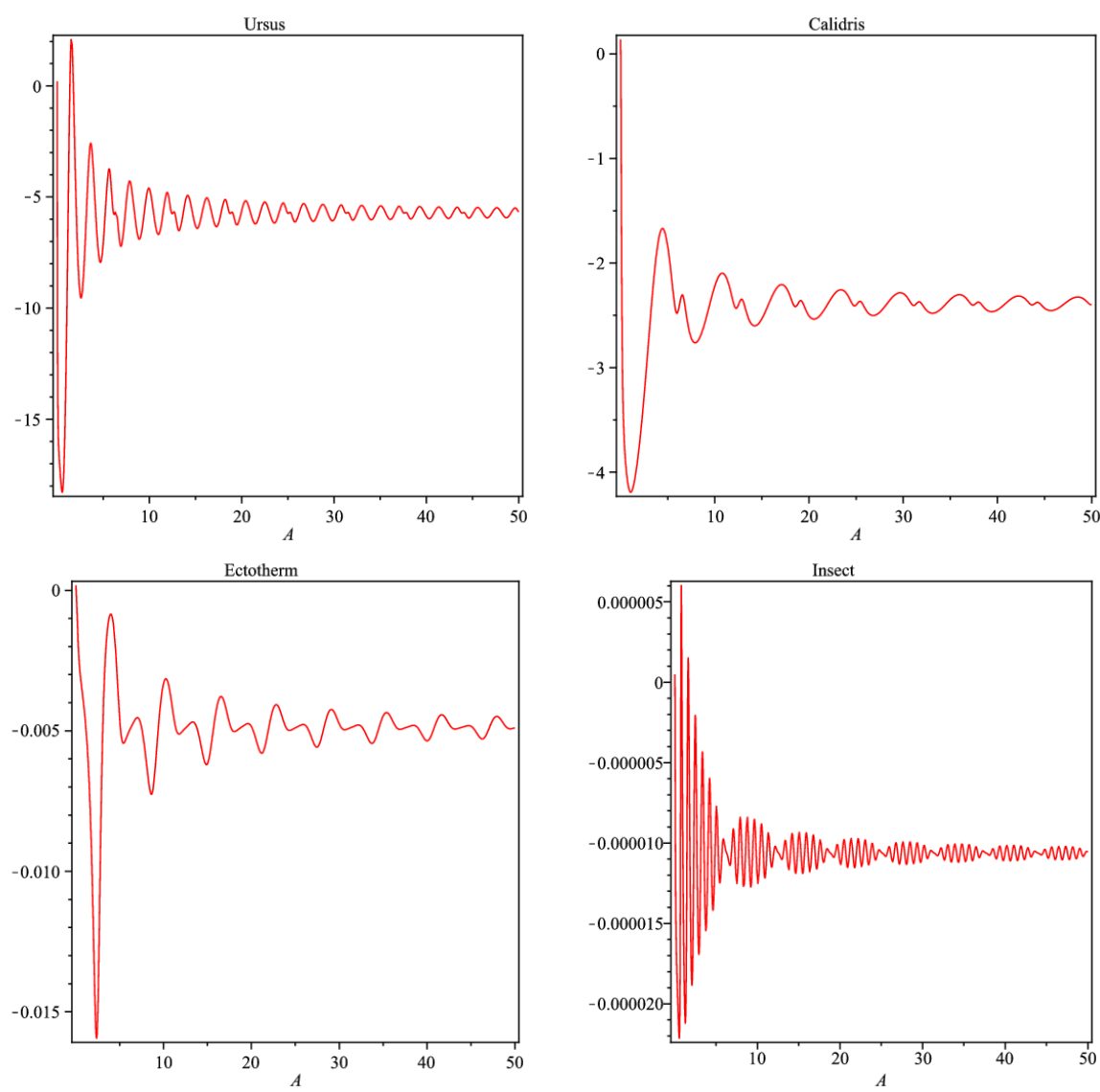

Figure 2: $k_{2}$ as a function of $A$

As a final remark, we would like to point out that our model was based on the assumption that competition occurs only within age-class. This is partially a technical assumption, although we found biological explanation for it. In a more realistic settings, one could use $\int_{a_{1}}^{a_{2}} \omega(a) L(a, t) d a$, where $\omega(a)$ is a weight function, instead of the function $L(a, t)$ to include competition between individuals of different age. 


\section{Age-structured population model in a spatio-temporally varying environment}

\subsection{Population in a patchy environment}

Until now, we have been dealing with age-structured populations in a temporally variable environment. Heterogeneity of the landscape and movement of individuals, although obvious, were neglected in the previous models. Thus, our aim is to include spatial structure into a model and to examine in what way this additional factor will influence persistence of a population.

In Section 2.4, we mentioned several different ways of handling spatial structure of the environment. In what follows, we will assume that habitat consists of $N$ patches. Different conditions on each patch give rise to a unique local subpopulation dynamics, which can be explained by model (4.1). Individuals disperse between patches, creating a complex dynamics of the whole system. Hence, the model we discus here is a generalization of the model presented in Paper II. Adding spatial heterogeneity brings a new level of complexity to the model, but it also gives a possibility to explain some natural phenomena and to propose strategies for management and conservation of species.

The population model is given by the following system of balance equations:

$$
\begin{aligned}
\frac{\partial n_{k}(a, t)}{\partial t}+\frac{\partial n_{k}(a, t)}{\partial a}= & -\mu_{k}(a, t) n_{k}(a, t)\left(1+\frac{n_{k}(a, t)}{L_{k}(a, t)}\right) \\
& +\sum_{j=1}^{N} D_{k j}(a, t) n_{j}(a, t), \quad 1 \leq k \leq N,
\end{aligned}
$$

where the functions $n_{k}(a, t)$ are defined for $a, t>0$ in the domain $\mathscr{B}$ given by

$$
\mathscr{B}:=\left\{(a, t) \in \mathbb{R}^{2}: 0<a<B(t), t>0\right\}
$$

and $B(t)>0$ is the time-dependent maximal length of life. The boundary and initial conditions are:

$$
\begin{aligned}
& n_{k}(0, t)=\int_{0}^{\infty} m_{k}(a, t) n_{k}(a, t) d a, \quad t>0, \\
& n_{k}(a, 0)=f_{k}(a), \quad a>0,
\end{aligned}
$$

where $n_{k}(a, t)$ is the number of individuals of age $a$ at time $t$ on patch $k, \mu_{k}(a, t)$ is the death rate, $L_{k}(a, t)$ the regulating function, $D_{k j}(a, t)$ dispersion coefficients, $m_{k}(a, t)$ the birth rate and $f_{k}(a)$ the initial distribution of population.

The regulating function $L_{k}(a, t)$ has the same function as it has in the model (4.1): it represents limitations imposed on individuals by the local environment and the logistic term $\frac{\mu_{k}(a, t) n_{k}^{2}(a, t)}{L_{k}(a, t)}$ describes density-dependent mortality on patch $k$.

Underlying assumptions considering the initial distribution of population are: 1) some patches may be empty at the initial time, and 2) in order for population to survive, sufficiently young individuals must occupy at least one patch. 
The dispersion coefficients $D_{k j}(a, t)$ satisfy the Metzler property

$$
D_{k j}(a, t) \geq 0, \quad k \neq j, \quad(a, t) \in \mathscr{B},
$$

and define a proportion of individuals of age $a$ at time $t$ on patch $j$ that migrates to patch $k$.

In the further analysis, we will use the fact that he dispersion matrix $D(a, t)$ can be related to a directed graph. Namely, any matrix $A$ that satisfies the Metzler property is associated to a directed graph (digraph) $\Gamma(A)$ with nodes labeled by $\{1,2, \ldots, N\}$, where an arc leads from $i$ to $j$ if and only if $A_{i j}>0$. We say that $j$ is reachable from $i$, if there exists a directed path from $i$ to $j$. A digraph is called connected from vertex $i$ if $j$ is reachable from $i$ for all $j \neq i$. A patch $k$ is accessible at age $a \geq 0$ if the associated digraph $\Gamma(D(a, t))$ is connected from $k$ for any $t>0$.

Population models that investigate source-sink dynamics rely on the fact that individuals can move from one patch to the other. This is especially important in modeling migrations, which are sometimes defined as a round-trip from the birthplace. Although there are isolated habitats in nature, we study patches that are connected, which explains introduction of accessibility of patches.

In order to obtain biologically meaningful model, we assume that $m_{k}, \mu_{k}, L_{k}, D_{k j}$ are continuous functions in $\overline{\mathscr{B}}$ and the following conditions hold:

(H1) there exists $0<b_{1}<b_{2}$ such that $b_{1} \leq B(t) \leq b_{2}$ for all $t \geq 0$ and

$$
\operatorname{Lip}(B):=\sup _{0<t_{1}<t_{2}<\infty} \frac{\left|B\left(t_{2}\right)-B\left(t_{1}\right)\right|}{\left|t_{2}-t_{1}\right|}<1
$$

(H2) each $m_{k}(a, t)$ is a uniformly bounded in $\overline{\mathscr{B}}$ and there exist $0<a_{m}<A_{m}<b_{1}$ such that for all $1 \leq k \leq N$

$$
\operatorname{supp} m_{k} \subset\left[a_{m}, A_{m}\right] \times \mathbb{R}^{+} ;
$$

(H3) all $\mu_{k}$ are uniformly bounded from below:

$$
\min _{1 \leq k \leq N} \inf _{(a, t) \in \mathscr{\mathscr { B }}} \mu_{k}(a, t)=\mu_{0}>0
$$

(H4) each $f_{k}(a)$ is a bounded continuous function and $\operatorname{supp} f_{k} \subset[0, B(0))$;

(H5) there exist constant $L_{0}>0$ such that $\frac{1}{L_{0}} \leq L_{k}(a, t) \leq L_{0}$ in $\mathscr{B}$;

By introducing function $\rho(t)=n(0, t)$ we can represent solution to (5.1)-(5.3) in the following way:

$$
n(a, t)= \begin{cases}\Phi(a, t-a ; \rho), & t>a, \\ \Psi(a, a-t ; f), & a \geq t,\end{cases}
$$


where function $\Phi(a, t ; \rho)$ solves (5.1) with the boundary condition $\Phi(0, t ; \rho)=\rho(t)$, and $\Psi(a, t ; f)$ solves $(5.1)$ with the initial condition $\Psi(a, 0 ; f)=f(a)$.

Moreover, the population problem (5.1)-(5.3) can be reduced to the following integral equation

$$
\rho(t)=\mathscr{K} \rho(t)+\mathscr{F} f(t),
$$

where the operators $\mathscr{K}$ and $\mathscr{F}$ are defined component-wise by:

$$
\begin{aligned}
& (\mathscr{K} \rho)_{k}(t)=\int_{0}^{t} m_{k}(a, t) \Phi_{k}(a, t-a ; \rho) d a, \\
& (\mathscr{F} f)_{k}(t)=\int_{t}^{\infty} m_{k}(a, t) \Psi_{k}(a, a-t ; f) d a .
\end{aligned}
$$

For given $f \in C\left(\mathbb{R}_{+}, \mathbb{R}_{+}^{N}\right)$, we prove existence and uniqueness of a solution to equation (5.6).

\subsection{Asymptotics in a constant environment}

Assumption that a population occupies temporally unchangeable patches implies that the structure parameters are time-independent functions. As we have already mentioned, the original problem (5.1)-(5.3) can be reduced to the integral equation (5.6). Our aim is to study large-time behavior of its solutions.

We begin by investigating stationary (time-independent) solutions to the equation (5.6). To this end, we define operator $\bar{K}: \mathbb{R}^{N} \rightarrow \mathbb{R}^{N}$ component-wise by the right-hand side of (5.2), i.e., by:

$$
(\bar{K} \rho)_{k}=\int_{0}^{\infty} m_{k}(a) \varphi_{k}(a ; \rho) d a
$$

and $\varphi_{k}(a ; \rho)$ is time-independent solution to (5.1) with the constant boundary condition $\varphi_{k}(0 ; \rho)=\rho_{k}, \rho=\left(\rho_{1}, \ldots, \rho_{N}\right)$.

Relation (5.2) for this solution reads as

$$
\rho=\overline{\mathscr{K}} \rho,
$$

and (5.9) will be called characteristic equation. Clearly, $\rho=0$ is always a solution, but our goal is to find out when a solution with positive components exists.

Another operator that will be useful in the study of solution to (5.6) is the net reproductive operator, denoted by $\mathscr{R}_{0}$. Like $\bar{K}$, the operator $\mathscr{R}_{0}$ is defined by the right hand side in (5.2), i.e., we have that

$$
\left(\mathscr{R}_{0} \rho\right)_{k}=\int_{0}^{\infty} m_{k}(a) \phi_{k}(a ; \rho) d a,
$$

where $\phi_{k}(a ; \rho)$ solves the linearized time-independent version of (5.1) (obtained by formally assuming that $\left.\frac{1}{L_{k}(a)} \equiv 0\right)$ with the constant boundary condition $\phi_{k}(0 ; \rho)=\rho_{k}$.

Let us suppose that 
(C1) Let us denote by $\bar{a}_{k}=\sup \left\{a: m_{k}(a)>0\right\}$ the maximal fertility age in population $k$. For any $k$ there exists $\beta_{k}<\bar{a}_{k}$ such that the patch $k$ is accessible at age $\beta_{k}$.

The condition (C1) has a biological interpretation: for any patch $k$ there exists a moment $\beta_{k}$ such that dispersal from any other patch $j$ to $k$ is possible within the reproductive period. Namely, according to biological research, there are many different reasons for dispersal, where response to the environmental conditions, prevention of inbreeding and competition for mates are some of them; see Bowler and Benton (2005). Thus, we can assume that dispersers and residents differ with respect to the life-history traits, genetics and demography. When it comes to the latter, young females in their reproductive age are often among dispersers, see, e.g., Gaines and McClenagha (1980) and Greenwood and Harvey (1982). Very old individuals usually do not undertake breeding dispersal, which is the topic of our study. This justifies the definition of $\beta_{k}$.

Under assumption (C1), we show that he operator $\mathscr{R}_{0}$ is strongly positive. Its spectral radius $\sigma\left(\mathscr{R}_{0}\right)$, called the net reproductive rate, is equal to its largest positive eigenvalue.

To motivate the definition of the operator $\mathscr{R}_{0}$, we consider a population which lives on a single patch. In this case, the net reproductive rate $R_{0}$ is defined by (3.8) and coincides with $\sigma\left(\mathscr{R}_{0}\right)$. The net reproductive operator $\mathscr{R}_{0}$ is then just a multiplication by $R_{0}$. Even if there are several isolated patches, every local subpopulation behaves accordingly to the value of the net reproductive rate on the respective patch.

If a population inhabits several patches connected by dispersal, $\mathscr{R}_{0}$ and $\sigma\left(\mathscr{R}_{0}\right)$ as we define them, represent a natural extension of the net reproductive operator and the net reproductive rate from one to several patches. Within this framework, we are able to determine under what conditions a nontrivial solution to the characteristic equation (5.9) exists. Namely, the following dichotomy holds:

- if $\sigma\left(\mathscr{R}_{0}\right) \leq 1$, then $\theta=0$ is the only solution to (5.9),

- if $\sigma\left(\mathscr{R}_{0}\right)>1$, then there exists a single solution $\theta$ to (5.9) and all its components are strictly positive.

However, solutions to equation (5.6) are not necessarily constant. Our analysis shows that any solution to (5.6) tends to $\theta$ at infinity, i.e., we have that

$$
\lim _{t \rightarrow \infty} \chi(t)=\theta
$$

Combining the previous results, we get characterization of an arbitrary solution to the equation (5.6):

- if $\sigma\left(\mathscr{R}_{0}\right) \leq 1$, then $\chi(t) \rightarrow 0$ as $t \rightarrow \infty$,

- if $\sigma\left(\mathscr{R}_{0}\right)>1$, then $\chi(t) \rightarrow \theta$ as $t \rightarrow \infty$ and all components of $\theta$ are strictly positive.

We have a similar result for the total population $v(t)$ :

- if $\sigma\left(\mathscr{R}_{0}\right) \leq 1$, then $v(t) \rightarrow 0$ as $t \rightarrow \infty$, 
- if $\sigma\left(\mathscr{R}_{0}\right)>1$, then $v(t) \rightarrow \int_{0}^{\infty} \varphi(a ; \theta) d a$ as $t \rightarrow \infty$,

where $\varphi(a ; \theta)$ is a solution to the time-independent problem (5.1) with $\theta$ as the initial condition and $\theta$ is the maximal solution to the characteristic equation.

In this way, the net reproductive rate $\sigma\left(\mathscr{R}_{0}\right)$ effectively determines permanency of a solution to the equation (5.6) and of the total population on $N$ constant patches. Here, as in the one-dimensional case, $\sigma\left(\mathscr{R}_{0}\right) \leq 1$ implies extinction of population on all patches, while $\sigma\left(\mathscr{R}_{0}\right)>1$ grants permanence of population.

\subsubsection{Two-side estimates for $\sigma\left(\mathscr{R}_{0}\right)$ and for the solution to (5.9)}

Both reproduction and migration are energetically costly processes for individuals. The trade-off between the two has been noted for many species, including migratory birds and some insects, see for instance Mole and Zera (1993), Schmidt-Wellenburg et al. (2008), Guerra (2011). This provides a biological justification for the assumption that individuals may die, but do not reproduce during migration. Mathematically, the fact that some individuals that are leaving patch $j$ can eventually die before reaching patch $k$, but cannot give birth is stated as:

$$
\sum_{k=1}^{N} D_{k j}(a) \leq\left|D_{j j}(a)\right|, \quad 1 \leq j \leq N .
$$

Using this fact, we obtain estimates from above and below for $\sigma\left(\mathscr{R}_{0}\right)$ and for a solution to the characteristic equation. Let

$$
\mu(a)=\min _{1 \leq k \leq N} \mu_{k}(a), \quad m(a)=\max _{1 \leq k \leq N} m_{k}(a), \quad L(a)=\max _{1 \leq k \leq N} L_{k}(a) .
$$

A two-side estimates for the net reproductive rate reads as:

$$
\begin{aligned}
\max _{1 \leq k \leq N} \int_{0}^{\infty} m_{k}(a) e^{-\int_{0}^{a}\left(\mu_{k}(v)+\left|D_{k k}(v)\right|\right) d v} d a & \leq \sigma\left(\mathscr{R}_{0}\right) \\
& \leq \int_{0}^{\infty} m(a) e^{-\int_{0}^{a} \mu(v) d v} d a,
\end{aligned}
$$

while an estimate for a solution $\rho$ of the equation (5.9) is as follows:

$$
\rho_{k}^{-} \leq \rho_{k} \leq \rho^{+},
$$

where $\rho_{k}^{-}$and $\rho^{+}$are solutions to equations

$$
\int_{0}^{\infty} \frac{m_{k}(a) e^{-\int_{0}^{a}\left(\mu_{k}(\nu)+\left|D_{k k}(v)\right|\right) d v}}{1+\rho_{k}^{-} \pi_{k}^{-}(a)} d a=1 \quad \text { and } \quad \int_{0}^{\infty} \frac{m(a) e^{-\int_{0}^{a} \mu(\nu) d v}}{1+\rho^{+} \pi(a)} d a=1
$$

with

$$
\pi_{k}^{-}(a)=\int_{0}^{a} \frac{\mu_{k}(\nu) e^{-\int_{0}^{v}\left(\mu_{k}(s)+\left|D_{k k}(s)\right|\right) d s}}{L_{k}(\nu)} d \nu \quad \text { and } \quad \pi(a)=\frac{1}{N} \int_{0}^{a} \frac{\mu(\nu)}{L(\nu)} e^{-\int_{0}^{v} \mu(s) d s} d \nu .
$$

Notice that the assumption (5.10) is needed only for derivation of the right hand side of inequality (5.11). 


\subsection{Asymptotics in a periodic environment}

Most often, natural habitats are changing periodically. Thus, the assumption that the model parameters are periodic functions with respect to time is reasonable. In studying large time behavior of solution to equation (5.6), a pivotal role belongs to the characteristic equation

$$
\rho(t)=\widetilde{\mathscr{K}} \rho(t),
$$

where the operator $\widetilde{\mathscr{K}}$ is given by the right hand side of (5.2) and $n_{k}(a, t)$ solves (5.1) with periodic boundary condition $n_{k}(0, t)=\rho_{k}(t)$.

The operator $\overline{\mathscr{K}}$ is absolutely continuous and preserves the cone

$$
C_{T}\left(\mathbb{R}_{+}, \mathbb{R}_{+}^{N}\right)=\left\{\rho \in C\left(\mathbb{R}_{+}, \mathbb{R}_{+}^{N}\right): \rho(t+T)=\rho(t)\right\} .
$$

Similarly to the time-independent case, we introduce the net reproductive operator $\widetilde{\mathscr{R}}_{0}$ by the right hand side of (5.2) assuming that $n_{k}(a, t)$ solves linearized (5.1), i.e., (5.1) without the nonlinear term, and with the periodic boundary condition $n_{k}(0, t)=\rho_{k}(t)$. In this case, we have that

$$
\widetilde{\mathscr{R}}_{0} \rho=\lim _{\Lambda \rightarrow 0} \frac{1}{\Lambda} \widetilde{\mathscr{K}} \Lambda \rho .
$$

In analogy to (C1), let us assume that the following holds:

(P1) For any $k$ there exists $\beta_{k}<\bar{a}_{k}$ such that the patch $k$ is accessible at age $\beta_{k}$, where $\bar{a}_{k}=\min _{t \geq 0} \sup \left\{a: m_{k}(a, t)>0\right\}$.

Under these assumptions, the operator $\widetilde{\mathscr{R}}_{0}$ is strictly positive, linear and defined on space of periodic continuous functions. Its spectral radius $\sigma\left(\widetilde{\mathscr{R}}_{0}\right)$ is equal to the largest eigenvalue and it is called the net reproductive rate.

One of the main results about the periodic case, as it was for the time-independent case, is the following dichotomy:

- if $\sigma\left(\widetilde{\mathscr{R}}_{0}\right) \leq 1$, then the equation (5.13) has only trivial solution $\theta \equiv 0$,

- if $\sigma\left(\widetilde{\mathscr{R}}_{0}\right)>1$, then the equation (5.13) has a single nontrivial solution $\theta(t)$ whose all components are positive.

Since an arbitrary solution to the equation (5.13) has $\theta$ as a limit at infinity, combining this with the previous result leads us to the following claim:

- if $\sigma\left(\widetilde{\mathscr{R}}_{0}\right) \leq 1$, then $\chi(t) \rightarrow 0$ as $t \rightarrow \infty$,

- if $\sigma\left(\widetilde{\mathscr{R}}_{0}\right)>1$, then $\chi(t) \rightarrow \theta(t)$ as $t \rightarrow \infty$.

As in the time-independent case, we extend analysis to the total population $v(t)$ and obtain:

- if $\sigma\left(\widetilde{\mathscr{R}}_{0}\right) \leq 1$, then $v(t) \rightarrow 0$ as $t \rightarrow \infty$, 
- if $\sigma\left(\widetilde{\mathscr{R}}_{0}\right)>1$, then $v(t) \rightarrow \int_{0}^{\infty} \Phi(a, t-a ; \theta) d a$ as $t \rightarrow \infty$,

where $\Phi(a, t ; \theta)$ is a solution to the original initial value problem and $\theta(t)$ is the maximal solution to the characteristic equation.

Thus, in the periodic as in the time-independent case, the net reproductive rate $\sigma\left(\widetilde{\mathscr{R}}_{0}\right)$ determines extinction or permanence of population on all patches.

\subsection{Two-side estimates in an irregular environment}

In the most general case when the environment is changing irregularly, large-time behavior of a solution to the original problem (5.1)-(5.3) can be examined using auxiliary periodic models. The vital rates, regulating function and dispersion coefficients can be bounded from above and below by periodic functions, thus allowing us to formulate two periodic models. One of them provides upper bounds for the original problem, and the other provides lower bounds. The number of newborns in the original problem, $\rho(t)$, is then estimated by the number of newborns in the associated periodic problems. In other words, the following holds:

$$
\theta^{-}(t)-\varepsilon \leq \rho(t) \leq \theta^{+}(t)+\varepsilon,
$$

where $\theta^{ \pm}(t)$ are solutions of the characteristic equations for periodic problems and $\varepsilon>0$ is a small number. Similar estimate is valid for the total population.

\subsection{Source-sink dynamics}

The fact that organisms move and that their dispersal is sometimes crucial for survival is neither new nor surprising. The research, both in ecology and mathematics, indicates that dispersal often improves population permanence. Mathematical models used for answering this kind of question are rarely fully age-structured, i.e., in most cases they presuppose two age-classes (young and adults). We will exploit source-sink dynamics, well known in ecology to explain permanence of age-structured populations in two different cases: when a population inhabits a single source and several sinks, or when a population inhabits only sink patches. For simplicity, we assume that the environment is constant and analyze the system

$$
\begin{aligned}
\frac{d \varphi_{k}(a ; \rho)}{d a} & =-\mu_{k}(a) \varphi_{k}(a ; \rho)+\sum_{j=1}^{N} D_{k j}(a) \varphi_{j}(a ; \rho), \quad a \geq 0, \\
\varphi_{k}(0 ; \rho) & =\rho_{k}, \quad 1 \leq k \leq N .
\end{aligned}
$$

\subsubsection{A single source and several sinks}

In order to explain the influence of dispersion on persistence of population when one of the patches is a source, we compare the system of $N$ patches where dispersal is possible with the system of $N$ isolated patches. We assume that the dispersion coefficients are given by $D_{k j}(a)=\varepsilon B_{k j}(a)$, for $\varepsilon \geq 0$ and the parameters $B_{k j}(a)$ satisfy condition (C1). 
The system of isolated patches corresponds to the problem (5.14) with $\varepsilon=0$ and $D_{k j}(a)=0$ for $a \geq 0$ and $1 \leq k, j \leq N$. The net reproductive rate on each patch is

$$
\sigma_{k}=\int_{0}^{\infty} m_{k}(a) e^{-\int_{0}^{a} \mu_{k}(v) d v} d a .
$$

Suppose that $\sigma_{1}>1$ and $\sigma_{k} \leq 1, k \geq 2$, and denote a positive solution to the population problem on patch $k$ by $\rho_{k}(t)$. Then, according to the analysis in Paper II, we have that

$$
\rho_{1}(t) \rightarrow \rho_{1}^{*} \text { and } \rho_{k}(t) \rightarrow 0 \text { as } t \rightarrow \infty, k \geq 2,
$$

where $\rho_{1}^{*}$ is a solution to the characteristic equation

$$
\int_{0}^{\infty} \frac{m_{1}(a) e^{-\int_{0}^{a} \mu_{1}(v) d v}}{1+\rho_{1}^{*}\left(1-e^{-\int_{0}^{a} \mu_{1}(v) d v}\right)} d a=1 .
$$

In other words, population is permanent on the patch with the net reproductive rate larger than one, and goes to extinction on all patches with the net reproductive rate equal or less than one.

Consider now that migration is possible and that $\sigma_{1}>1$ and $\sigma_{k} \leq 1$ for $k \geq 2$ still hold. Solving system (5.14) leads us to the net reproductive operator

$$
\begin{aligned}
\left(\mathscr{R}_{0} \rho\right)_{k}= & \sigma_{k} \rho_{k} \\
& +\varepsilon \int_{0}^{\infty} m_{k}(a) e^{-\int_{0}^{a} \mu_{k}(v) d v} \int_{0}^{a} \sum_{j=1}^{N} \rho_{j} B_{k j}(s) e^{\int_{0}^{s}\left(\mu_{k}(v)-\mu_{j}(v)\right) d v} d s d a \\
& +O\left(\varepsilon^{2}\right) .
\end{aligned}
$$

Its largest eigenvalue is

$$
\sigma\left(\mathscr{R}_{0}\right)=\sigma_{1}+\varepsilon \int_{0}^{\infty} m_{1}(a) e^{-\int_{0}^{a} \mu_{1}(v) d v} B_{11}(a) d a+O\left(\varepsilon^{2}\right),
$$

and this is greater than one for small $\varepsilon>0$, provided that $B_{11}(a) \leq 0$ and strictly negative at least in one point of the support of $m_{1}$. This implies survival of population on all patches if emigration from the source is sufficiently small.

\subsubsection{Sinks without a source}

Jansen and Yoshimura (1998) used a simple model to analyze connection between population growth and allocation of the offspring on different patches. They commented that with a suitable allocation, survival of population is possible even in the case when all patches are sinks. We will demonstrate that this is possible for age-structured populations.

A realistic example of this type of behavior are populations of migratory birds. They have two habitats: breeding range and non-breeding range. The first is characterized by the high birth rate in summer and high death rate in winter. The latter has low birth and 
death rates. Thus, the breeding range is a sink because of the winter conditions, and the non-breeding range is a sink because of too few births.

In the following example, we simplify the situation by assuming that the environment is constant. Let the death rates $\mu_{1}$ and $\mu_{2}$ be constant on support of $m_{1}$ and $m_{2}$, respectively. In addition, suppose that $\mu_{1}>\mu_{2}>0$ and that the net reproductive rates on each patch satisfy

$$
\sigma_{k}=\int_{0}^{\infty} m_{k}(a) e^{-\mu_{k} a} d a=1, \quad k=1,2 .
$$

This implies extinction of the population on both patches if there is no dispersal. On the first patch, extinction is caused by the high death rate and on the second patch, the cause is low birth rate.

Suppose now that migration is possible and the dispersion matrix $D$ in (5.14) satisfies

$$
D=\varepsilon\left(\begin{array}{cc}
-1 & 1 \\
1 & -1
\end{array}\right), \quad \varepsilon>0 \text {. }
$$

Solving system (5.14) for $N=2$ implies that the net reproductive operator satisfies

$$
\left(\mathscr{R}_{0} \rho\right)_{k}=\rho_{k}+\varepsilon \int_{0}^{\infty} m_{k}(a) e^{-\mu_{k} a} h_{k}(a) d a+O\left(\varepsilon^{2}\right), \quad k=1,2 .
$$

This can be written as

$$
\mathscr{R}_{0} \rho=\rho+\varepsilon \mathscr{P} \rho+O\left(\varepsilon^{2} \rho\right)
$$

where the operator $\mathscr{P}$ depends on the vital rates. Thus, to show that $\sigma\left(\mathscr{R}_{0}\right)>1$, it is sufficient to show that $\mathscr{P} \rho>0$ for some choice of the vital rates and vector $\rho$.

In Paper IV, we show that for the appropriate choice of $m_{k}$ and $\mu_{k}$ for $k=1,2$, one can find $\rho$ such that $\mathscr{P} \rho>0$ and hence, the corresponding net reproductive rate will be greater than one. This also proves permanence of the population on both patches.

\subsubsection{Extinction on all patches}

A big part of research is dedicated to investigation under what conditions a population is permanent. However, for practical purposes, such as pest control or management of species, one needs to know what can cause extinction of a population. This question becomes especially important if individuals move between habitats.

Namely, if a population lives on $N$ isolated patches, it can either survive or go to extinction on each of them, regardless of population dynamics on other patches. This means that there are $2^{N}$ possible outcomes for the whole system.

If the patches are connected by dispersal, due to the rescue effect and recolonization, a local extinction of the species can be amended. This implies that every local subpopulations contribute to a uniquely defined dynamics for the whole system. Therefore, causing extinction of a subpopulation on a single patch does not have to be a permanent. 
As we have seen, extinction on all patches occurs if $\sigma\left(\mathscr{R}_{0}\right) \leq 1$. Let us recall a very crude estimate for the net reproductive rate given by (5.11):

$$
\sigma\left(\mathscr{R}_{0}\right) \leq \int_{0}^{\infty} m(a) e^{-\int_{0}^{a} \mu(v) d v} d a
$$

where $m(a)$ is the highest fertility on all patches and $\mu(a)$ is the lowest mortality on all patches. What this estimate suggests is that the global extinction is imminent if the lowest mortality so high (of if the highest fertility is so low) that the value of the previous integral becomes less than one. Notice that this estimate holds regardless of the dispersion pattern, and according to this, satisfying management of species would rely on changing vital rates.

On the other hand, according to (5.15), a specific choice of a dispersion pattern promotes permanence. If the goal is to cause the global extinction, choosing a different dispersion pattern might lead to it. In particular, making emigration from a source sufficiently large to cause extinction of population on the source, might lead to a collapse of the whole system. 


\section{Discussion}

As we have seen, qualitative analysis of solutions to the linear and logistic age-structured time-independent models involved solving characteristic equations (3.6) and (4.5), respectively. Population dynamics in both cases is determined by the vital rates and predicted by the net reproductive rate $R_{0}$. In the linear case, $R_{0} \geq 1$ means that the population is permanent, and $R_{0}<1$ implies extinction. On the other hand, in the logistic model, the population is permanent only for $R_{0}>1$ and faces extinction for $R_{0} \leq 1$.

Inclusion of temporal variability brings time-dependent vital rates and regulating function, and in turn, more complicated characteristic equations. In Paper I and Paper II, we define two auxiliary time-independent problems to obtain upper and lower bounds for a solution to the original problem. Taking the supremum of fecundity and survival, we define the time-independent best case, which provides upper bounds for the original problem. On the other hand, the infimum of fecundity and survival lead us to the time-independent worst case and lower bounds. Estimation of the population growth or decline in the original problem is determined by the value of the net reproductive rate in the two extreme situations.

Periodically changing environment attracts special interest due to the fact that most natural habitats exhibit some temporal change in conditions. Tuljapurkar (1985) used a linear matrix model to show that oscillations with period close to the generation time are beneficial for a population. Our analysis shows that the population response to the environmental change depends on the life history and on the frequency. This indicates that life-history should not be ignored in population models. The exemptions are very low frequencies, which are detrimental for all species in the linear model and beneficial for all species in the logistic model.

According to Paper I, the dominant role in determining asymptotic behavior of a solution play the transient vital rates. The only case when the frequency of oscillation determines population growth in the linear model is for $R_{0}=1$. However, the type of effect that environmental variability has depends on the species, which points out the importance of studying the combination of age-structure, time-dependency and life history.

In the logistic model, temporal variability can cause extinction if a population is very small and the amplitude of oscillation is sufficiently large. This is similar to the findings of Ripa and Lundberg (1996). For majority of frequencies, effects of oscillations on population growth are detrimental, which can be related to the observations made by Roughgarden (1975), Roughgarden (1979) and May (1973) that fluctuations of the environment keep population numbers below the carrying capacity.

The final step in modeling population growth is introducing spatial heterogeneity in the model. It is done through the assumption that population lives on $N$ patches connected by dispersal. Although it brings a new level of mathematical complexity, it also gives a possibility to explain some natural phenomena.

If the patches are isolated, local subpopulations are either permanent or go to extinction. This allows $2^{N}$ different outcomes for the dynamics of the whole system. If all patches are connected by dispersal, local dynamics contribute to a uniquely determined 
dynamics of the whole population. To describe global dynamics, instead of the original system of balance equations, we use its linearized counterpart. The net reproductive rate $R_{0}$ is substituted by the spectral radius of the net reproductive operator $\sigma\left(\mathscr{R}_{0}\right)$. Its role is to predict the global dynamics. Namely, if $\sigma\left(\mathscr{R}_{0}\right)>1$, a population is permanent on all patches. Otherwise, it faces global extinction.

The obvious improvement of this model in comparison to the linear and logistic models is the presence of dispersal. If suitably chosen, it can explain global permanence of population. We consider the situation when at least one patch is a source. The key role in this case plays the subpopulation on the source. In particular, dispersal of individuals that are able to reproduce needs to be limited, because their excessive emigration can cause the extinction of the local source subpopulation.

Another, more extreme, case of population permanence is when all patches are sinks. In this case, survival is possible for slowly declining populations, i.e., $\sigma_{k}=1$ for $1 \leq k \leq N$ and it depends on the pattern of the migration. Hastings and Botsfor (2006) argues that survival of some populations in a patchy environment depends on their return home, which indicates the importance of migration patterns. We demonstrate that survival on two sinks connected by small migration is possible for a certain choice of vital rates.

Dispersal between patches can be used for management of pest species. Namely, we showed that global extinction occurs if the combination of the highest birth rate and the lowest death rate on all patches produces $R_{0} \leq 1$. The other method for causing extinction on all patches is to destabilize source patches by extensive emigration of individuals in their reproductive age. These conclusions suggest what practical actions can be taken to ensure a successful pest control.

A common method for asymptotic analysis of a solution in all included papers is using upper and lower bounds. The choice of this method has both mathematical and ecological motivation. Namely, addition of different ecological factors made models more complex in the mathematical terms. As a consequence, the characteristic equations gradually become more difficult to solve. In order to overcome these difficulties, we formulate and analytically solve two time-independent auxiliary problems. Their solutions are used to define time-dependent upper and lower bounds of a solution to the original problem.

Ecologically, it is important to know evolution of the system, and not just exact number of individuals at any time. Upper and lower bounds are used to predict population dynamics in cases when we cannot have exact solution to the population problem. Namely, low upper bound may indicate extinction risk, while high lower bound may suggest population persistence. The advantage of having time-dependent bounds is that they allow deeper analysis of the factors that influence population growth. This can be, for example, a relation between the frequency of oscillation, life history and age structure, as we have seen in Paper II, or influence of dispersal on population permanence, like we had in Paper III and Paper IV. 


\subsection{Further research}

In Paper II, we considered intra-specific competition which provides negative feedback for population growth. However, density dependent factors are not necessarily detrimental for population growth. The Allee effect describes positive effects of increased density when density level is low. In most models that include the Allee effect, agestructure in either absent or it is presented by discrete age-classes. Incorporating the Allee effect in a continuous age-structured time-dependent model may reveal connections between different factors and their influence on population dynamics. Continuing this line of thought, it may be useful to investigate how age-dependent dispersion affects permanence of population, when local density-dependence is described by the Allee effect.

On the other hand, models that investigate relations between two or more species, rarely incorporate continuous age structure. Since predation, foraging capabilities, hiding form predators or group defense may depend on age, valuable insight into food web dynamics can be gained from a model that would include age structure. 


\section{Bibliography}

L. Allen. Persistence and extinction in single-species reaction-diffusion models. Bulletin of Mathematical Biology, 45:209-227, 1983.

O. N. Bjørnstad and B. T. Grenfell. Noisy clockwork: Time series analysis of population fluctuations in animals. Science Translational Medicine, 293 (5530):638-643, 2001. doi: $10.1126 /$ science.1062226.

D. E. Bowler and T. G. Benton. Causes and consequences of animal dispersal strategies: relating individual behaviour to spatial dynamics. Biol. Rev., 80:205-225, 2005.

M. S. Boyce and D. J. Daley. Population tracking of fluctuating environments and natural selection for tracking ability. Am. Nat., 115:480-491, 1980.

J. Chi and L. Chen. The effect of diffusion on the time varying logistic population growth. Computers Math. Applic., 36:1-9, 1998.

J. Chi and L. Chen. Permanence and extinction in logistic and lotka-volterra systems with diffusion. Journal of Mathematical Analysis and Applications, 258:512-535, 2001.

M. Chipot. On the equations of age-dependent population dynamics. Arch. Rational Mech. Anal., 82(1):13-25, 1983.

B. D. Coleman. Nonautonomous logistic equations as models of the adjustment of populations to environmental change. Math. Biosci., 45:159-173, 1979.

L. M. Cook. Oscillation in the simple logistic growth model. Nature, 1967.

T. Coulson, P. Rohani, and M. Pascual. Skeletons, noise and population growth: the end of an old debate? Trends in Ecology and Evolution, 19(7), 2004.

J. M. Cushing. Oscillatory population growth in periodic environments. Theor. Pop. Biol., 30:289-308, 1986a.

J. M. Cushing. Periodic mckendrick equations for age-structured population growth. Comp and Maths with App., 12A:513-526, 1986b.

P. C. Dias. Sources and sinks in population biology. Trends in Ecology and Evolution, 11: 326-330, 1996.

R. Elderkin. Nonlinear, globally age-dependent population models: Some basic theory. Journal of Mathematical Analysis and Applications, 108:546-562, 1985.

W. Feller. On the integral equation of renewal theory. Ann. Math. Stat., 12:243-267, 1941.

M. S. Gaines and L. R. McClenagha. Dispersal in small mammals. Annual Review of Ecology and Systematics, 11:163-196, 1980. 
P. J. Greenwood and P. H. Harvey. The natal and breeding dispersal of birds. Annual Review of Ecology and Systematics, 13:1-21, 1982.

P. A. Guerra. Evaluating the life-history trade-off between dispersal capability and reproduction in wing dimorphic insects: a meta-analysis. Biological Reviews, 86:813-835, 2011.

M. E. Gurtin and R. C. MacCamy. Nonlinear age-dependent population dynamics. Arch. Rat. Mech. Anal., 54:281-300, 1974.

A. Hastings. Complex interactions between dispersal and dynamics: Lessons from coupled logistic equations. Ecology, 44:1362-1372, 1993.

A. Hastings and L. W. Botsfor. Persistence of spatial populations depends on returning home. PNAS, 103:6067-6072, 2006.

M. Iannelli. Mathematical theory of age-structured population dynamics. Applied mathematics monographs 7. Giardini editori e stampatori, 1995. ISBN 8842702501,9788842702504 .

M. Iannelli and A. Pugliese. An introduction to mathematical population dynamics, volume 79 of Unitext. Springer, Cham, 2014. ISBN 978-3-319-03025-8; 978-3-31903026-5. doi: 10.1007/978-3-319-03026-5. URL http://dx.doi.org/10.1007/ 978-3-319-03026-5. Along the trail of Volterra and Lotka, La Matematica per il 3+2.

V. A. A. Jansen and J. Yoshimura. Populations can persist in an environment consisting of sink habitats only. Proc. Natl. Acad. Sci. USA Ecology, 95:3696-3698, 1998.

P. Kareiva and U. Wennergren. Connecting landscape patterns to ecosystem and population processes. Nature, 373:299-302, 1995.

M. Kot. Elements of mathematical ecology. Cambridge University Press, 2001.

P. Marcati. Some considerations on the mathematical approach to nonlinear age dependent population dynamics. Computers \& Mathematics with Applications, 9(3): 361-370, 1983.

R. May. Stability and complexity in model ecosystems. Princeton University Press, Princeton, N.Y., 1973.

A. G. McKendrick. Application of mathematics to medical problems, volume 44 of Proceedings of Edinburgh Mathematical Society. 1926.

S. Mole and A. Zera. Differential allocation of resources underlies the dispersalreproduction trade-off in the wing-dimorphic cricket, gryllus rubens. Oecologia, 93: 121-127, 1993.

D. Price. Carrying capacity reconsidered. Population and Environment, 21:5-26, 1999. 
H. R. Pulliam. Sources, sinks, and population regulation. American Naturalist, 132(5): 652-661, 1988.

J. Ripa and P. Lundberg. Noise colour and the risk of population extinctions. Proc. R. Soc. Lond., 263:1751-1753, 1996.

J. Roughgarden. A simple model for population dynamics in stochastic environments. Am. Nat., 109:713-736, 1975.

J. Roughgarden. Theory of Population Genetics and Evolutionary Ecology: An Introduction. Macmillan Publishing Co. Inc., New York, Collier Macmillan Publishers London, 1979.

C. A. Schmidt-Wellenburg, G. H. Visserb, B. Biebacha, K. Delheyd, M. Oltroggea, A. Wittenzellnere, H. Biebacha, and B. Kempenaers. Trade-off between migration and reproduction: does a high workload affect body condition and reproductive state? Behavioral Ecology, doi:10.1093/beheco/arn066, 2008.

F.R. Sharpe and A. J. Lotka. A problem in age distribution. Philosophical Magazine, 21: 435-438, 1911.

J. W.-H. So, J. Wu, and X. Zou. Structured population on two patches: modeling dispersal and delay. J. Math. Biol., 43:37-51, 2001.

J. H. Steele. A comparison of terrestrial and marine ecological systems. Nature, 313: 355-358, 1985.

N. C. Stenseth, A. Mysterud, G. Ottersen, J. W. Hurrell, K. S. Chan, and M. Lima. Ecological effects of climate fluctuations. Science, 297:1292-1296, 2002.

Y. Takeuchi. Diffusion effect on stability of lotka-volterra models. Bul. Math. Bio., 48: 585-601, 1986a.

Y. Takeuchi. Global stability in generalized lotka-volterra diffusion systems. Journal of Math. Analysis and Appl., 116:209-221, 1986b.

A. Terry. Dynamics of structured population on two patches. Journal of Mathematical Analysis and Applications, 378:1-15, 2011.

S. Tuljapurkar. Population dynamics in variable environments vi. cyclical environments. Theo. Pop. Bio., 28:1-17, 1985.

R. R. Vance. The effect of dispersal on population stability in one-species, discrete-space population growth models. The American Naturalist, 123:230-254, 1984.

H. von Foerster. Some remarks on changing populations. The Kinetics of Cellular Proliferation, F. Stohlman, Jr, editor. Grune \& Straton, New York, pages 382-407, 1959. 
G. F. Webb. Theory of nonlinear age-dependent population dynamics. Marcel Dekker Inc, 1985.

G. F. Webb. Logistic model of structured population growth. Comp. and Maths. with Appl., 12A:527-539, 1986.

P. Weng, C. Xiao, and X. Zou. Rich dynamics in a non-local population model over three patches. Nonlinear Dyn., 59:161-172, 2010.

U. Wennergren, M. Ruckelshaus, and P. Kareiva. The promise and limitations of spatial models in conservation biology. Oikos, 74:349-356, 1995.

E. Zeidler. Nonlinear functional analysis and its applications I. Springer-Verlag, 1986. 


\section{Included papers}





\section{Papers}

The articles associated with this thesis have been removed for copyright reasons. For more details about these see:

http://urn.kb.se/resolve?urn=urn:nbn:se:liu:diva-130927 